\title{
Spaces of directed paths on pre-cubical sets II
}

\author{
Krzysztof Ziemiański ${ }^{1}$
}

Received: 13 January 2019 / Accepted: 20 August 2019 / Published online: 30 August 2019 (c) The Author(s) 2019

\section{Abstract}

For a given pre-cubical set ( $\square$-set) $K$ with two distinguished vertices $\mathbf{0}, \mathbf{1}$, we prove that the space $\vec{P}(K)_{\mathbf{0}}^{\mathbf{1}}$ of d-paths on the geometric realization of $K$ with source $\mathbf{0}$ and target $\mathbf{1}$ is homotopy equivalent to its subspace $\vec{P}^{t}(K)_{\mathbf{0}}^{\mathbf{1}}$ of tame d-paths. When $K$ is the underlying $\square$-set of a Higher Dimensional Automaton $A$, tame d-paths on $K$ represent step executions of $A$. Then, we define the cube chain category of $K$ and prove that its nerve is weakly homotopy equivalent to $\vec{P}(K)_{\mathbf{0}}^{\mathbf{1}}$.

Keywords Directed path space · Pre-cubical set · Higher Dimensional Automaton · Synchronization · Tame path · Homotopy colimit

Mathematics Subject Classification 55P15 - 55P35 - 68N30 - 68Q85

\section{Introduction}

A directed space, or a d-space (Grandis 2003), is a topological space $X$ with a distinguished family of paths $\vec{P}(X)$, called d-paths, that contains all constant paths and is closed with respect to concatenations and non-decreasing reparametrizations. Directed spaces serve as models in concurrency: points of a given directed space represent possible states of a concurrent program, while d-paths represent possible partial executions. It is important to know the homotopy type of the space $\vec{P}(X)_{x}^{y}$ of d-paths beginning at the point $x$ and ending at the point $y$. If $x$ and $y$ are the initial and the final state of the program, respectively, it represents the "execution space" of the program modeled by $X$. Also, calculation of some invariants of d-spaces, e.g. component categories

The author thanks the Hausdorff Research Institute for Mathematics for its hospitality during his visit in Bonn as a part of the programme Applied and Computational Algebraic Topology in September, 2017. The author also thanks the referees for many valuable comments and remarks.

Krzysztof Ziemiański

ziemians@mimuw.edu.pl

1 Faculty of Mathematics, Informatics and Mechanics, University of Warsaw, ul. Banacha 2, 02-097 Warsaw, Poland 

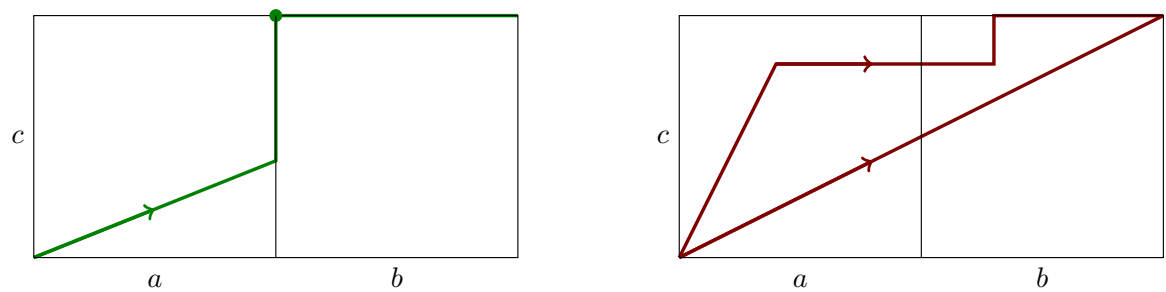

Fig. 1 The d-path in the left-hand picture is tame; it represents an execution which can be divided into two steps: in the first, $a$ and $c$ are performed and, in the second, $b$ is performed. No such division is possible for the d-paths in the right-hand picture

(Raussen 2007; Ziemiański 2019b) and natural homology (Dubut et al. 2015), requires knowledge of the homotopy types of d-path spaces between two particular points.

In this paper, we consider this problem for $\mathrm{d}$-spaces that are geometric realizations of pre-cubical sets, called also $\square$-sets. $\square$-sets play an important role in concurrency: Higher Dimensional Automata introduced by Pratt (1991) are $\square$-sets equipped with a labeling of edges, and then executions of a Higher Dimensional Automaton can be interpreted as d-paths on the geometric realization of the underlying $\square$-set. van Glabbeek (2006) has shown that many other models for concurrency (e.g. Petri nets) can be translated to Higher Dimensional Automata and, therefore, to $\square$-sets.

The problem of calculating the homotopy types of d-path spaces between two vertices of a $\square$-set was studied in several papers, e.g. Raussen $(2010,2012)$ and Ziemiański (2017, 2019a). All these results work only for special classes of $\square$-sets, like Euclidean complexes or proper $\square$-sets, i.e., those whose triangulations are simplicial complexes. There are some interesting examples of $\square$-sets that do not fall into any of these classes; notably, the universal labeling $\square$-sets ! $\Sigma$ introduced by Goubault (2002), see also Fahrenberg and Legay (2013). In this paper, we consider $\square$-sets in their full generality.

For an arbitrary $\square$-set $K$ with two distinguished vertices $\mathbf{0}$, 1, we prove that the space of d-paths $\vec{P}(|K|)_{\mathbf{0}}^{\mathbf{1}}$ with source $\mathbf{0}$ and target $\mathbf{1}$ is homotopy equivalent to its subspace $\vec{P}^{t}(|K|)_{\mathbf{0}}^{\mathbf{1}}$ of tame d-paths (Theorem 6.1). A d-path is tame if it can be divided into segments each of which runs from the initial to the final vertex of some cube (see Fig. 1). Then we define the cube chain category of $K$, denoted $\mathrm{Ch}(K)$, and prove that the geometric realization of the nerve of $\mathrm{Ch}(K)$ is weakly homotopy equivalent to the space of tame d-paths on $K$ (Theorem 7.5). This provides a combinatorial model for the execution space of $K$ (Theorem 7.6), which can be used for explicit calculations of its homotopy type. All these constructions are functorial with respect to $K$, regarded as an object in the category of bi-pointed $\square$-sets. These theorems generalize the results of Ziemiański (2017).

Theorem 6.1 is interesting in itself. In terms of Higher Dimensional Automata, tame d-paths represent synchronized executions: at every step, a number of processes performs one complete step while the others remain idle. As a consequence of Theorem 6.1, not only homotopy classes of synchronized executions of a given Higher Dimensional Automaton are the same as homotopy classes of all executions but also the respective execution spaces are homotopy equivalent. Thus, one may expect that all 
phenomena concerning executions of Higher Dimensional Automata can be observed as well after restricting to synchronized executions only.

Organization of the paper and relationship with Ziemiański (2017). The paper consists of two parts. The main goal of the first part (Sects. 3-6) is to prove the tamification theorem (Theorem 6.1), that of the second part (Sects. 7-10) is to prove Theorems 7.5 and 7.6. The general outline of this paper resembles that of Ziemiański (2017) but we need to use more subtle arguments here.

Fix a bi-pointed $\square$-set $K$ with initial vertex $\mathbf{0}$ and final vertex 1. All d-paths $\alpha \in$ $\vec{P}(K)_{0}^{1}$ have integral $L^{1}$-lengths (Raussen 2009), and the spaces of d-paths having length $n$, denoted $\vec{P}(K ; n)_{\mathbf{0}}^{\mathbf{1}}$, will be handled separately for every $n \in \mathbb{Z}_{\geq 0}$.

In Sect. 3 we define tracks, which are sequences of cubes such that for two subsequent cubes, some upper face of the preceding cube is a lower face of the succeeding one. Tracks were investigated by Fahrenberg and Legay (2013); they called them cube paths. Then we prove that every d-path $\alpha \in \vec{P}(K)_{\mathbf{0}}^{\mathbf{1}}$ is a concatenation of d-paths contained in consecutive cubes of some track. In Sect. 4 , we define the set of actions that correspond to a given track $\mathfrak{C}$. In Sect. 5, we introduce progress functions of tracks and investigate the relationship between progress functions of a track $\mathfrak{C}$ and d-paths contained in $\mathfrak{C}$. Then in Sect. 6 , we construct, in a functorial way, a self-map of the space $\vec{P}(K ; n)_{\mathbf{0}}^{\mathbf{1}}$ that is homotopic to the identity map and maps all natural d-paths (i.e., parametrized by length) into tame d-paths. The proof of the latter statement makes essential use of progress functions. Since the space of natural d-paths is homotopy equivalent to the space of all d-paths, this implies Theorem 6.1.

This argument is essentially different from the one used in the proof of the tamification theorem in the previous paper (Ziemiański 2017, Theorem 5.6). That one follows from a similar result for d-simplicial complexes (Ziemiański 2012), which was proved by constructing a certain self-deformation of a given d-simplicial complex. This selfdeformation was given by a direct but complicated and non-functorial formula and it is not clear how to interpret it in terms of concurrent processes. While a general outline of the argument is similar, the tamification via progress functions is more intuitive: a d-path $\alpha$ contained in a track $\mathfrak{C}$ is deformed to a tame d-path by "speeding up" the executions of the actions of $\mathfrak{C}$.

In the second part, we introduce cube chains: sequences of cubes in $K$ such that the final vertex of the preceding cube is the initial vertex of the succeeding one. They constitute a special case of tracks. Then, we formulate the main result of the second part of the paper stating that the nerve of the category $\mathrm{Ch}(K)$ of cube chains on $K$ is weakly homotopy equivalent to the space of natural tame d-paths on $K$ (Theorem 7.5). A natural d-path $\alpha \in \vec{N}(K)_{\mathbf{0}}^{\mathbf{1}}$ is tame if and only it is contained in some cube chain, i.e., it admits a presentation as a concatenation of d-paths contained in consecutive cubes of a given cube chain $\mathbf{c}$. Such a presentation is called a natural tame presentation of $\alpha$; the difficulty that arises here is due to the fact that $\alpha$ may have many natural tame presentations in c. This is essentially more complicated than the situation considered in Ziemiański (2017), where there is a good cover of the space of natural tame d-paths $\vec{N}^{t}(K)_{0}^{\mathbf{1}}$ indexed by the poset of cube chains on $K$, and the analogue of Theorem 7.5 can be proven using the Nerve Lemma. 
Instead of a good cover, we need to investigate the functor $G:=\vec{N}_{[0, n]}\left(\square^{\vee^{(-)}}\right)_{\mathbf{0}}^{\mathbf{1}}$ : $\mathrm{Ch}(K) \rightarrow$ Top, where $G(\mathbf{c})$ is the space of natural tame presentations in c, equipped with a map $F_{n}^{K}:$ colim $G \rightarrow \vec{N}_{[0, n]}^{t}(K)_{\mathbf{0}}^{\mathbf{1}}$. In Sect. 8, we investigate properties of natural tame presentations. In Sects. 9 and 10, we apply the results of Sect. 8 to prove that the middle and the right-hand map in the sequence (7.5)

$$
|\mathrm{Ch}(K)| \longleftarrow \text { hocolim } G \longrightarrow \operatorname{colim} G \stackrel{F_{n}^{K}}{\longrightarrow} \vec{N}_{[0, n]}^{t}(K)_{\mathbf{0}}^{\mathbf{1}}
$$

are weak homotopy equivalences. This is easy for the left-hand map, so Theorem 7.5 follows.

\section{Preliminaries}

In this section we recall definitions and introduce notation that is used later on. See Fajstrup et al. (2006) or Fajstrup et al. (2016) for surveys which cover most of these topics.

\section{1 d-spaces}

Grandis (2003) defines a d-space as a pair $(X, \vec{P}(X))$, where $X$ is a topological space and $\vec{P}(X)$ is a family of paths on $X$ (with domain $[0,1]$ ) that contains all constant paths and which is closed with respect to concatenations and non-decreasing reparametrizations. In this paper, it is more convenient to use a slightly different, though equivalent, definition. $A d$-space is a topological space $X$ equipped with a d-structure. $A d$-structure on $X$ is a collection of families of paths $\left\{\vec{P}_{[a, b]}(X)\right\}_{a<b \in \mathbb{R}}$,

$$
\vec{P}_{[a, b]}(X) \subseteq P_{[a, b]}(X):=\operatorname{map}([a, b], X),
$$

called d-paths, such that

- every constant path const $t_{[a, b]}^{x}:[a, b] \ni t \mapsto x \in X$ is a d-path,

- for every non-decreasing function $f:[a, b] \rightarrow[c, d]$ and every d-path $\alpha \in$ $\vec{P}_{[c, d]}(X)$, the path $\alpha \circ f$ is a d-path, i.e., $\alpha \circ f \in \vec{P}_{[a, b]}(X)$,

- if $a<b<c$ and $\alpha \in \vec{P}_{[a, b]}(X), \beta \in \vec{P}_{[b, c]}(X)$ are d-paths such that $\alpha(b)=\beta(b)$, then the concatenation of $\alpha$ and $\beta$ :

$$
(\alpha * \beta)(t)= \begin{cases}\alpha(t) & \text { for } t \in[a, b] \\ \beta(t) & \text { for } t \in[b, c] .\end{cases}
$$

is a d-path, i.e., $\alpha * \beta \in \vec{P}_{[a, c]}(X)$.

This definition is equivalent to the original definition by Grandis: if $\left(X,\left\{\vec{P}_{[a, b]}(X)\right\}_{a<b}\right)$ is a d-space, then $\left(X, \vec{P}_{[0,1]}(X)\right)$ is a d-space in Grandis' sense. If $(X, \vec{P}(X))$ is a dspace in Grandis' sense, then by letting

$$
\vec{P}_{[a, b]}(X)=\{[a, b] \ni t \mapsto \alpha((t-a) /(b-a)) \in X \mid \alpha \in \vec{P}(X)\}
$$


we obtain the d-space as defined above. We will occasionally write $\vec{P}(X)$ for $\vec{P}_{[0,1]}(X)$.

The sets $\vec{P}_{[a, b]}(X)$ are topological spaces, with the compact-open topology inherited from the space of all paths $P_{[a, b]}(X)=\operatorname{map}([a, b], X)$.

Given two d-spaces $X, Y$, a continuous map $f: X \rightarrow Y$ is $a d$-map if $f \circ \alpha \in$ $\vec{P}_{[a, b]}(Y)$ for every d-path $\alpha \in \vec{P}_{[a, b]}(X)$ and for all $a<b \in \mathbb{R}$. The family of d-spaces with d-maps forms the category dTop, which is complete and cocomplete.

For a d-space $X$ and $x, y \in X$, denote by $\vec{P}_{[a, b]}(X)_{x}^{y} \subseteq \vec{P}_{[a, b]}(X)$ the subspace of d-paths $\alpha$ such that $\alpha(a)=x$ and $\alpha(b)=y$.

A bi-pointed $d$-space is a d-space $X$ with two distinguished points: an initial point $\mathbf{0}_{X}$ and a final point $\mathbf{1}_{X} \in X$. A bi-pointed d-map is a d-map that preserves the initial and the final points. The category of bi-pointed d-spaces and bi-pointed maps will be denoted by dTop**

\subsection{Directed intervals and cubes}

Let $s<t \in \mathbb{R}$. The directed interval is the d-space $\overrightarrow{[s, t]}$ such that $\vec{P}_{[a, b]}(\overrightarrow{[s, t]})$ is the space of non-decreasing continuous functions $[a, b] \rightarrow[s, t]$. The d-space $\vec{I}=\overrightarrow{[0,1]}$ will be called the directed unit interval.

The directed $n$-cube $\vec{I}^{n}$ is the categorical product of $n$ copies of the directed unit interval. A path on $\vec{I}^{n}$ is a d-path if all its coordinates are d-paths in $\vec{I}$, i.e., they are nondecreasing functions. Points of $\vec{I}^{n}$ will be denoted by bold letters, and their coordinates are distinguished by upper indices, so that, for example $\mathbf{x}=\left(x^{1}, x^{2}, \ldots, x^{n}\right) \in \vec{I}^{n}$. A similar convention will be used for d-paths: for $\beta \in \vec{P}\left(\vec{I}^{n}\right), \beta^{i} \in \vec{P}(\vec{I})$ denotes the $i$ th coordinate of $\beta$. We will write $|\mathbf{x}|$ for $\sum_{i=1}^{n} x^{i}$.

Whenever $\vec{I}$ or $\vec{I}^{n}$ are considered as bi-pointed d-spaces, their initial and final points are $0,1 \in \vec{I}$ and $\mathbf{0}=(0, \ldots, 0), \mathbf{1}=(1, \ldots, 1) \in \vec{I}^{n}$, respectively.

\subsection{Quotient d-spaces}

Let $X, Y$ be topological spaces and let $p: X \rightarrow Y$ be a quotient map. Assume that $X$ is equipped with a d-structure $\left\{\vec{P}_{[a, b]}(X)\right\}_{a<b \in \mathbb{R}}$. The quotient d-structure on the space $Y$ is defined in the following way: a path $\alpha \in P_{[a, b]}(Y)$ is a d-path if and only if there exist numbers $a=t_{0}<\cdots<t_{n}=b$ and d-paths $\beta_{i} \in \vec{P}_{\left[t_{i-1}, t_{i}\right]}(X)$ such that $\alpha(t)=p\left(\beta_{i}(t)\right)$ for $t \in\left[t_{i-1}, t_{i}\right]$. The quotient d-structure is the smallest d-structure on $Y$ such that $p$ is a d-map. The space $Y$ with this quotient d-structure will be called the quotient $d$-space of $X$.

\section{$2.4 \square$-sets}

A pre-cubical set, or $a \square$-set $K$ is a sequence of disjoint sets $(K[n])_{n \geq 0}$ with a collection of face maps $\left(d_{i}^{\varepsilon}: K[n] \rightarrow K[n-1]\right)$ for $n>0, \varepsilon \in\{0,1\}, i \in\{1, \ldots, n\}$ such that $d_{i}^{\varepsilon} d_{j}^{\eta}=d_{j-1}^{\eta} d_{i}^{\varepsilon}$ for all $\varepsilon, \eta \in\{0,1\}$ and $i<j$. Elements of the sets $K[n]$ will be called $n$--cubes or just cubes and 0 -cubes will be called vertices. The dimension of a cube $c$ is the integer $\operatorname{dim}(c)$ such that $c \in K[\operatorname{dim}(c)]$. For $\square$-sets $K, L, a \square$-map 
$f: K \rightarrow L$ is a sequence of maps $f[n]: K[n] \rightarrow L[n]$ that commute with the face maps. The category of $\square$-sets and $\square$-maps will be denoted by $\square$ Set.

An example of a $\square$-set is the standard $n$-cube $\square^{n}$, such that $\square^{n}[k]$ is the set of functions $\{1, \ldots, n\} \rightarrow\{0,1, *\}$ that take value $*$ for exactly $k$ arguments. The face map $d_{i}^{\varepsilon}$ converts the $i$ th occurrence of $*$ into $\varepsilon$. The only element of $\square^{n}[n]$ will be denoted by $u_{n}$. For any $\square$-set $K$, there is a $1-1$ correspondence between the set of $n$-cubes $K[n]$ and the set of $\square$-maps $\square^{n} \rightarrow K$ : for every $c \in K[n]$ there exists a unique map $f_{c}: \square^{n} \rightarrow K$ such that $f_{c}\left(u_{n}\right)=c$.

The $k$ th skeleton of a $\square$-set $K$ is the sub- $\square$-set $K_{(k)} \subseteq K$ given by

$$
K_{(k)}[n]= \begin{cases}K[n] & \text { for } n \leq k \\ \varnothing & \text { for } n>k\end{cases}
$$

The boundary of the standard $n$-cube is $\partial \square^{n}:=\square_{(n-1)}^{n}$.

Given a $\square$-set $K$, a subset $A=\left\{a_{1}<\cdots<a_{k}\right\} \subseteq\{1, \ldots, n\}$ and $\varepsilon \in\{0,1\}$, define the iterated face map

$$
d_{A}^{\varepsilon}:=d_{a_{1}}^{\varepsilon} \circ d_{a_{2}}^{\varepsilon} \circ \cdots \circ d_{a_{k}}^{\varepsilon}: K[n] \rightarrow K[n-k] .
$$

Denote $d^{\varepsilon}:=d_{\{1, \ldots, n\}}^{\varepsilon}: K[n] \rightarrow K[0]$. For a cube $c \in K[n], d^{0}(c)$ and $d^{1}(c)$ will be called the initial and the final vertex of $c$, respectively.

A bi-pointed $\square$-set is a triple $\left(K, \mathbf{0}_{K}, \mathbf{1}_{k}\right)$, where $K$ is a $\square$-set and $\mathbf{0}_{K}, \mathbf{1}_{K} \in K[0]$ are distinguished vertices; we will write $\mathbf{0}$ and $\mathbf{1}$ for $\mathbf{0}_{K}$ and $\mathbf{1}_{K}$ whenever this does not lead to confusion. The category of bi-pointed $\square$-sets and base-points-preserving $\square$-maps will be denoted by $\square$ Set $_{*}^{*}$.

\subsection{Geometric realization}

Let $K$ be a $\square$-set. The geometric realization of $K$ is the quotient d-space

$$
|K|=\left(\coprod_{n \geq 0} K[n] \times \vec{I}^{n}\right) / \sim .
$$

The relation $\sim$ is generated by $\left(c, \delta_{i}^{\varepsilon}(\mathbf{x})\right) \sim\left(d_{i}^{\varepsilon}(c), \mathbf{x}\right)$ for all $n \geq 1, i \in\{1, \ldots, n\}$, $\varepsilon \in\{0,1\}, c \in K[n]$ and $\mathbf{x} \in \vec{I}^{n-1}$, where

$$
\delta_{i}^{\varepsilon}: \vec{I}^{n-1} \ni\left(x^{1}, \ldots, x^{n-1}\right) \mapsto\left(x^{1}, \ldots, x^{i-1}, \varepsilon, x^{i}, \ldots, x^{n-1}\right) \in \vec{I}^{n}
$$

is the coface map. For $c \in K[n]$ and $\mathbf{x}=\left(x^{1}, \ldots, x^{n}\right) \in \vec{I}^{n},[c ; \mathbf{x}] \in|K|$ denotes the point represented by $(c, \mathbf{x})$. Every point $p \in|K|$ admits a unique canonical presentation $p=\left[c_{p} ; \mathbf{x}_{p}\right]$ such that $x_{p}^{j} \neq 0,1$ for all $j$. The cube $c_{p}$ will be called the carrier of $p$. Each presentation of $p$ has the form

$$
p=\left[c^{\prime}, \delta_{i_{1}}^{\varepsilon_{1}}\left(\ldots\left(\delta_{i_{r}}^{\varepsilon_{r}}\left(\mathbf{x}_{p}\right)\right) \ldots\right)\right]
$$


where $c^{\prime} \in K\left[\operatorname{dim}\left(c_{p}\right)+r\right]$ is a cube such that $d_{i_{1}}^{\varepsilon_{1}}\left(\ldots\left(d_{i_{r}}^{\varepsilon_{r}}\left(c^{\prime}\right)\right) \ldots\right)=c_{p}$.

For $A=\left\{a_{1}<\cdots<a_{k}\right\} \subseteq\{1, \ldots, n\}$ and $\varepsilon \in\{0,1\}$, the iterated coface map is defined by the formula

$$
\delta_{A}^{\varepsilon}:=\delta_{a_{k}}^{\varepsilon} \circ \delta_{a_{k-1}}^{\varepsilon} \circ \cdots \circ \delta_{a_{1}}^{\varepsilon}: \vec{I}^{n-k} \rightarrow \vec{I}^{n}
$$

Notice that if

$$
\bar{A}=\left\{\bar{a}_{1}<\cdots<\bar{a}_{n-k}\right\}=\{1, \ldots, n\} \backslash A,
$$

and $\mathbf{x}=\left(x^{1}, \ldots, x^{n-k}\right) \in \vec{I}^{n-k}$, then

$$
\delta_{A}^{\varepsilon}(\mathbf{x})^{i}= \begin{cases}\varepsilon & \text { for } i \in A \\ x^{j} & \text { for } i=\bar{a}_{j} \in \bar{A} .\end{cases}
$$

Also, we have $\left[d_{A}^{\varepsilon}(c) ; \mathbf{x}\right]=\left[c ; \delta_{A}^{\varepsilon}(\mathbf{x})\right]$ for all $c, \mathbf{x}$ and $A$.

Every $\square$-map $f: K \rightarrow L$ induces the d-map

$$
|f|:|K| \ni[c ; \mathbf{x}] \mapsto[f(c) ; \mathbf{x}] \in|L|
$$

Thus, the geometric realization defines the functors $|-|: \square$ Set $\rightarrow$ dTop and $|-|$ : $\square$ Set $_{*}^{*} \rightarrow$ dTop $_{*}^{*}$.

We will usually skip the vertical bars and write $\vec{P}(K)$ for $\vec{P}(|K|)$.

\subsection{Presentations of d-paths}

Let $K$ be a $\square$-set. A presentation of a d-path $\alpha \in \vec{P}_{[a, b]}(|K|)$ consists of

- a sequence $\left(c_{i}\right)_{i=1}^{l}$ of cubes of $K$, and

- a sequence $\left(\beta_{i} \in \vec{P}_{\left[t_{i-1}, t_{i}\right]}\left(\vec{I}^{\operatorname{dim}\left(c_{i}\right)}\right)\right)_{i=1}^{l}$ of d-paths,

such that

- $a=t_{0} \leq t_{1} \leq \cdots \leq t_{l-1} \leq t_{l}=b$, and

- $\alpha(t)=\left[c_{i} ; \beta_{i}(t)\right]$ for every $i \in\{1, \ldots, l\}$ and $t \in\left[t_{i-1}, t_{i}\right]$.

We write such a presentation as

$$
\alpha={ }^{t_{0}}\left[c_{1} ; \beta_{1}\right] \stackrel{t_{1}}{*}\left[c_{2} ; \beta_{2}\right] \stackrel{t_{2}}{*} \cdots{ }^{t_{l-1}} *\left[c_{l} ; \beta_{l}\right]^{t_{l}}
$$

It follows immediately that every d-path in $|K|$ admits a presentation.

\subsection{Length}

Let $K$ be a $\square$-set. The $L^{1}$-length, or just the length of a d-path $\alpha \in \vec{P}_{[a, b]}(K)$ is defined as

$$
\operatorname{len}(\alpha)=\sum_{i=1}^{l}\left|\beta_{i}\left(t_{i}\right)\right|-\left|\beta_{i}\left(t_{i-1}\right)\right|=\sum_{i=1}^{l} \sum_{j=1}^{\operatorname{dim}\left(c_{i}\right)} \beta_{i}^{j}\left(t_{i}\right)-\beta_{i}^{j}\left(t_{i-1}\right),
$$


for some presentation (2.7) of $\alpha$. The length was introduced by Raussen (2009, Section 2). This definition does not depend on the choice of a presentation, and defines, for every $a<b \in \mathbb{R}$, a continuous function len : $\vec{P}(K)_{[a, b]} \rightarrow \mathbb{R}_{\geq 0}$ (Raussen 2009, Proposition 2.7). If $x, y \in|K|$ and d-paths $\alpha, \alpha^{\prime} \in \vec{P}_{[a, b]}(K)_{x}^{y}$ are d-homotopic (i.e., they are contained in the same path-connected component of $\left.\vec{P}_{[a, b]}(K)_{x}^{y}\right)$ then $\operatorname{len}(\alpha)=\operatorname{len}\left(\alpha^{\prime}\right)$. Furthermore, if $K$ is a bi-pointed $\square$-set, then the length of every d-path $\alpha \in \vec{P}_{[a, b]}(K)_{\mathbf{0}}^{\mathbf{1}}$ is an integer. As a consequence, there is a decomposition

$$
\vec{P}_{[a, b]}(K)_{\mathbf{0}}^{\mathbf{1}} \cong \coprod_{n \geq 0} \vec{P}_{[a, b]}(K ; n)_{\mathbf{0}}^{\mathbf{1}}
$$

where $\vec{P}_{[a, b]}(K ; n)_{\mathbf{0}}^{\mathbf{1}}$ stands for the space of d-paths having length $n$.

\subsection{Naturalization}

We say that a d-path $\alpha \in \vec{P}_{[a, b]}(K)$ is natural if len $\left(\left.\alpha\right|_{[c, d]}\right)=d-c$ for every $a \leq c \leq d \leq b$. Let $\vec{N}_{[a, b]}(K) \subseteq \vec{P}_{[a, b]}(K)$ denote the subspace of natural d-paths.

Natural d-paths were introduced and studied by Raussen (2009). He proved that for every d-path $\alpha \in \vec{P}_{[a, b]}(K)$ there exists a unique natural d-path nat $(\alpha) \in \vec{N}_{[0, \operatorname{len}(\alpha)]}(K)$ such that

$$
\alpha(t)=\operatorname{nat}(\alpha)\left(\operatorname{len}\left(\left.\alpha\right|_{[a, t]}\right)\right) .
$$

Furthermore, for a bi-pointed $\square$-set $K$, the map

$$
\operatorname{nat}_{n}^{K}: \vec{P}_{[0, n]}(K ; n)_{\mathbf{0}}^{\mathbf{1}} \ni \alpha \mapsto \operatorname{nat}(\alpha) \in \vec{N}_{[0, n]}(K)_{\mathbf{0}}^{\mathbf{1}}
$$

is a homotopy inverse of the inclusion map (Raussen 2009, Propositions 2.15 and 2.16). The map $\operatorname{nat}_{n}^{K}$ is functorial with respect to $K \in \square \mathbf{S e t}_{*}^{*}$.

\subsection{Tame paths}

A d-path $\alpha \in \vec{P}_{[a, b]}(K)_{\mathbf{0}}^{\mathbf{1}}$ is tame if it admits a presentation (2.7) such that, for every $i \in\{1, \ldots, l-1\}, \alpha\left(t_{i}\right)$ is a vertex, i.e., has the form [ $v$; ()] for some $v \in K[0]$. This definition generalizes the earlier definitions for d-paths on d-simplicial complexes (Ziemiański 2012) and on proper $\square$-sets (Ziemiański 2017).

If $\alpha$ is tame, then one can impose an even stronger condition on its presentation. For every $i$, all coordinates of $\beta_{i}\left(t_{i}\right)$ are either 0 or 1 ; if $\beta_{i}^{j}\left(t_{i}\right)=0$, then $\beta_{i}^{j}(t)=0$ for all $t \in\left[t_{i-1}, t_{i}\right]$. Hence the segment $\left[c_{i}, \beta_{i}\right]$ may be replaced by $\left[d_{j}^{0}\left(c_{i}\right), \beta_{i}^{\prime}\right]$, where $\beta_{i}^{\prime}$ is the path obtained from $\beta_{i}$ by skipping its $j$ th coordinate. By repeating this operation, we obtain a presentation such that $\beta_{i}\left(t_{i}\right)=(1, \ldots, 1)$ for all $i$. In a similar way, we can guarantee that $\beta_{i}\left(t_{i-1}\right)=(0, \ldots, 0)$. A presentation $(2.7)$ such that $\beta_{i}\left(t_{i-1}\right)=\mathbf{0}$ and $\beta_{i}\left(t_{i}\right)=\mathbf{1}$ for all $i$ will be called a tame presentation of $\alpha$.

Let $\vec{P}_{[a, b]}^{t}(K)_{\mathbf{0}}^{\mathbf{1}}$ (resp. $\left.\vec{N}_{[a, b]}^{t}(K)_{\mathbf{0}}^{\mathbf{1}}\right)$ denote the space of all tame (resp. natural tame) d-paths on $K$ from $\mathbf{0}$ to $\mathbf{1}$. 


\section{Tracks}

Let $K$ be a bi-pointed $\square$-set. Every d-path $\alpha \in \vec{P}(K)_{\mathbf{0}}^{\mathbf{1}}$ is a concatenation of d-paths having the form $[c ; \beta]$, where $c$ is a cube of $K$ and $\beta$ is a d-path in $\vec{I}^{\operatorname{dim}(c)}$. We will show that we can impose extra conditions on the cubes and the paths which appear in such a presentation.

Definition 3.1 A track $\mathfrak{C}$ in $K$ is a sequence of triples $\left(c_{i}, A_{i}, B_{i}\right)_{i=1}^{l}$, where

- $c_{1}, \ldots, c_{l}$ are cubes of $K$,

- $A_{i}, B_{i} \subseteq\left\{1, \ldots, \operatorname{dim}\left(c_{i}\right)\right\}$

such that

(a) $d_{A_{1}}^{0}\left(c_{1}\right)=\mathbf{0}_{K}$,

(b) $d_{B_{l}}^{1}\left(c_{l}\right)=\mathbf{1}_{K}$,

(c) $d_{B_{i}}^{1}\left(c_{i}\right)=d_{A_{i+1}}^{0}\left(c_{i+1}\right)$ for every $i \in\{1, \ldots, l-1\}$.

(d) the sets $A_{1}, B_{l}$ and $B_{i} \cup A_{i+1}$ are non-empty.

Remark Tracks are equivalent to cube paths introduced by Fahrenberg and Legay (2013, Section 3).

Proposition 3.2 For every track $\mathfrak{C}=\left(c_{i}, A_{i}, B_{i}\right)_{i=1}^{l}$ we have $\sum_{i=1}^{l}\left|A_{i}\right|=\sum_{i=1}^{l}\left|B_{i}\right|$.

Proof. Conditions (a), (b) and (c) imply that $\operatorname{dim}\left(c_{1}\right)=\left|A_{1}\right|, \operatorname{dim}\left(c_{i+1}\right)=\operatorname{dim}\left(c_{i}\right)-$ $\left|B_{i}\right|+\left|A_{i+1}\right|$ and $\operatorname{dim}\left(c_{l}\right)=\left|B_{l}\right|$. We have

$$
\left|B_{l}\right|=\operatorname{dim}\left(c_{l}\right)=\operatorname{dim}\left(c_{1}\right)+\sum_{i=2}^{l}\left|A_{i}\right|-\sum_{i=1}^{l-1}\left|B_{i}\right|=\sum_{i=1}^{l}\left|A_{i}\right|-\sum_{i=1}^{l-1}\left|B_{i}\right| .
$$

The integer $\sum\left|A_{i}\right|=\sum\left|B_{i}\right|$ will be called the length of the track $\mathfrak{C}$ and denoted len $(\mathfrak{C})$.

Definition 3.3 Let $\mathfrak{C}=\left(c_{i}, A_{i}, B_{i}\right)_{i=1}^{l}$ be a track in $K$ and let $\alpha \in \vec{P}_{[a, b]}(K)_{\mathbf{0}}^{\mathbf{1}}$. Denote $e_{i}=d_{B_{i}}^{1}\left(c_{i}\right)=d_{A_{i+1}}^{0}\left(c_{i+1}\right)$. A presentation

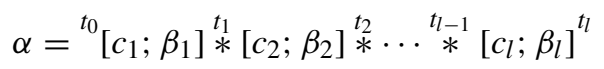

is $a \mathfrak{C}$-presentation of $\alpha$ if there exist points $\mathbf{x}_{i} \in \vec{I}^{\operatorname{dim}\left(e_{i}\right)}, i \in\{1, \ldots, l-1\}$ such that for all $i$ :

(1) $\beta_{i}\left(t_{i}\right)=\delta_{B_{i}}^{1}\left(\mathbf{x}_{i}\right)$,

(2) $\beta_{i+1}\left(t_{i}\right)=\delta_{A_{i+1}}^{0}\left(\mathbf{x}_{i}\right)$.

We say that $\alpha$ is contained in $\mathfrak{C}$ if it admits a $\mathfrak{C}$-presentation. The space of d-paths contained in $\mathfrak{C}$ will be denoted by $\vec{P}_{[a, b]}(K, \mathfrak{C})$.

Proposition 3.4 If $\alpha \in \vec{P}_{[a, b]}(K ; \mathfrak{C})$, then len $(\alpha)=\operatorname{len}(\mathfrak{C})$. 
Proof. Choose a $\mathfrak{C}$-presentation of $\alpha$, as in Definition 3.3. For every $i \in\{1, \ldots, l\}$ we have

$$
\operatorname{len}\left(\left.\alpha\right|_{\left[t_{i-1}, t_{i}\right]}\right)=\operatorname{len}\left(\beta_{i}\right)=\left|\beta_{i}\left(t_{i}\right)\right|-\left|\beta_{i}\left(t_{i-1}\right)\right|=\left|B_{i}\right|+\left|\mathbf{x}_{i}\right|-\left|\mathbf{x}_{i-1}\right|
$$

when assuming $\left|\mathbf{x}_{0}\right|=\left|\mathbf{x}_{l}\right|=0$. Thus,

$$
\operatorname{len}(\alpha)=\sum_{i=1}^{l} \operatorname{len}\left(\left.\alpha\right|_{\left[t_{i-1}, t_{i}\right]}\right)=\sum_{i=1}^{l}\left|B_{i}\right|=\operatorname{len}(\mathfrak{C})
$$

Fajstrup proved (Fajstrup 2005, 2.20) that if $K$ is a geometric $\square$-set (Fajstrup 2005, 2.8) then every d-path $\alpha \in \vec{P}(K)_{\mathbf{0}}^{\mathbf{1}}$ is contained in a track called the carrier sequence of $\alpha$. Below we prove an analogue of this result for arbitrary $\square$-sets.

Proposition 3.5 Every non-constant d-path $\alpha \in \vec{P}_{[a, b]}(K)_{\mathbf{0}}^{\mathbf{1}}$ is contained in some track.

Proof Choose a presentation

$$
\alpha={ }^{t_{0}}\left[c_{1} ; \beta_{1}\right] \stackrel{t_{1}}{*}\left[c_{2} ; \beta_{2}\right] \stackrel{t_{2}}{*} \cdot{ }^{t_{l-1}} *\left[c_{l} ; \beta_{l}\right]^{t_{l}} .
$$

such that the integer $l+\sum_{i=1}^{l} \operatorname{dim}\left(c_{i}\right)$ is minimal among all presentations of $\alpha$. Then:

(1) $\beta_{i}^{j}\left(t_{i}\right)>0$ for all $i \in\{1, \ldots, l\}, j \in\left\{1, \ldots, \operatorname{dim}\left(c_{i}\right)\right\}$. Otherwise, there exist $i, j$ such that $\beta_{i}^{j}\left(t_{i}\right)=0$, which implies that $\beta_{i}^{j}(t)=0$ for all $t \in\left[t_{i-1}, t_{i}\right]$. Hence, the segment $\left[c_{i}, \beta_{i}\right]$ may be replaced by

$$
\left[d_{j}^{0}\left(c_{i}\right),\left(\beta_{i}^{1}, \ldots, \beta_{i}^{j-1}, \beta_{i}^{j+1}, \ldots, \beta_{i}^{\operatorname{dim}\left(c_{i}\right)}\right)\right],
$$

which contradicts the assumption that $l+\sum \operatorname{dim}\left(c_{i}\right)$ is minimal.

(2) $\beta_{i}^{j}\left(t_{i-1}\right)<1$ for all $i \in\{1, \ldots, l\}, j \in\left\{1, \ldots, \operatorname{dim}\left(c_{i}\right)\right\}$; the argument is similar.

(3) For every $i \in\{1, \ldots, l-1\}$, there exists $j \in\left\{1, \ldots, \operatorname{dim}\left(c_{i}\right)\right\}$ such that $\beta_{i}^{j}\left(t_{i}\right)=1$, or there exists $k \in\left\{1, \ldots, \operatorname{dim}\left(c_{i+1}\right)\right\}$ such that $\beta_{i+1}^{k}\left(t_{i}\right)=0$. Assume otherwise; since $0<\beta_{i}^{j}\left(t_{i}\right), \beta_{i+1}^{k}\left(t_{i}\right)<1$ for all $j, k,\left[c_{i}, \beta_{i}\left(t_{i}\right)\right]$ and $\left[c_{i+1}, \beta_{i+1}\left(t_{i}\right)\right]$ are both canonical presentations of the point $\alpha\left(t_{i}\right)$. Thus, $c_{i}=c_{i+1}, \beta_{i}\left(t_{i}\right)=\beta_{i+1}\left(t_{i}\right)$ and the two segments $\left[c_{i} ; \beta_{i}\right]$ and $\left[c_{i+1} ; \beta_{i+1}\right]$ may be replaced by the single segment $\left[c_{i} ; \beta_{i} * \beta_{i+1}\right]$.

(4) $\operatorname{dim}\left(c_{1}\right), \operatorname{dim}\left(c_{l}\right)>0$. Otherwise the segment $\left[c_{1} ; \beta_{1}\right]$ (resp. $\left[c_{l}, \beta_{l}\right]$ ) could be merged with $\left[c_{2} ; \beta_{2}\right]$ (resp. $\left.\left[c_{l-1}, \beta_{l-1}\right]\right)$, which exists since $\alpha$ is not constant.

Let

$$
\begin{aligned}
& A_{i}=\left\{j \in\left\{1, \ldots, \operatorname{dim}\left(c_{i}\right)\right\} \mid \beta_{i}^{j}\left(t_{i-1}\right)=0\right\} \\
& B_{i}=\left\{j \in\left\{1, \ldots, \operatorname{dim}\left(c_{i}\right)\right\} \mid \beta_{i}^{j}\left(t_{i}\right)=1\right\} .
\end{aligned}
$$


We will check that $\mathfrak{C}=\left(c_{i}, A_{i}, B_{i}\right)_{i=1}^{l}$ is a track. We have $\mathbf{0}_{K}=\left[c_{1} ; \beta_{1}(a)\right]$ and $\beta_{i}^{j}(a)<1$ for all $j$; thus, $\beta_{i}^{j}(a)=0$ (since $\alpha\left(t_{0}\right)=\mathbf{0}_{K}$ is a vertex). Therefore, $A_{1}=$ $\left\{1, \ldots, \operatorname{dim}\left(c_{1}\right)\right\}$ and $d_{A_{1}}^{0}\left(c_{1}\right)=\mathbf{0}_{K}$. Similarly, $B_{l}=\left\{1, \ldots, \operatorname{dim}\left(c_{l}\right)\right\}$ and $d_{B_{l}}^{1}\left(c_{l}\right)=$ $\mathbf{1}_{K}$. Thus, conditions (a) and (b) of Definition 3.1 are satisfied. Condition 3.1.(d) follows from (3). It follows from (1) and (2) that, for every $i$, there exist:

- a unique $\mathbf{x}_{i} \in \vec{I}^{\operatorname{dim}\left(c_{i}\right)-\left|B_{i}\right|}$ such that $\delta_{B_{i}}^{1}\left(\mathbf{x}_{i}\right)=\beta_{i}\left(t_{i}\right)$, and

- a unique $\mathbf{y}_{i} \in \vec{I}^{\operatorname{dim}\left(c_{i+1}\right)-\left|A_{i+1}\right|}$ such that $\delta_{A_{i+1}}^{0}\left(\mathbf{y}_{i}\right)=\beta_{i+1}\left(t_{i}\right)$.

We have

$$
\begin{aligned}
& \alpha\left(t_{i}\right)=\left[c_{i} ; \beta_{i}\left(t_{i}\right)\right]=\left[c_{i} ; \delta_{B_{i}}^{1}\left(\mathbf{x}_{i}\right)\right]=\left[d_{B_{i}}^{1}\left(c_{i}\right) ; \mathbf{x}_{i}\right] \\
& \alpha\left(t_{i}\right)=\left[c_{i+1} ; \beta_{i+1}\left(t_{i}\right)\right]=\left[c_{i+1} ; \delta_{A_{i+1}}^{0}\left(\mathbf{y}_{i}\right)\right]=\left[d_{A_{i+1}}^{0}\left(c_{i+1}\right) ; \mathbf{y}_{i}\right] .
\end{aligned}
$$

All coordinates of both $\mathbf{x}_{i}$ and $\mathbf{y}_{i}$ are different from both 0 and 1 . Thus, $\left[d_{B_{i}}^{1}\left(c_{i}\right) ; \mathbf{x}_{i}\right]$ and $\left[d_{A_{i+1}}^{0}\left(c_{i+1}\right) ; \mathbf{y}_{i}\right]$ are both canonical presentations of the same point and, therefore, they are equal. As a consequence, $d_{B_{i}}^{1}\left(c_{i}\right)=d_{A_{i+1}}^{0}\left(c_{i+1}\right)$, which proves that Definition 3.1. (c) is satisfied. Thus, $\mathfrak{C}$ is a track. Moreover, the points $\mathbf{x}_{i}=\mathbf{y}_{i}$ fit into the Definition 3.3; hence, $\alpha$ is contained in $\mathfrak{C}$.

\section{Actions}

Every d-path $\alpha$ between vertices of a $\square$-set $K$ having length $n$ can be interpreted as a performance of $n$ different actions. This is an easy observation if $K$ is a Euclidean complex in the sense of Raussen and Ziemiański (2014). In this section we will show how to interpret actions when $K$ is an arbitrary $\square$-set.

Fix a bi-pointed $\square$-set $K$ and a track $\mathfrak{C}=\left(c_{i}, A_{i}, B_{i}\right)_{i=1}^{l}$ in $K$ having length $n$. For $i \in\{1, \ldots, l\}$, denote

$$
\begin{aligned}
q_{i} & =\operatorname{dim}\left(c_{i}\right)-\left|B_{i}\right|=\operatorname{dim}\left(c_{i+1}\right)-\left|A_{i+1}\right|, \\
\bar{A}_{i} & =\left\{\bar{a}_{i}^{1}<\bar{a}_{i}^{2}<\cdots<\bar{a}_{i}^{q_{i-1}}\right\}=\left\{1, \ldots, \operatorname{dim}\left(c_{i}\right)\right\} \backslash A_{i}, \\
\bar{B}_{i} & =\left\{\bar{b}_{i}^{1}<\bar{b}_{i}^{2}<\cdots<\bar{b}_{i}^{q_{i}}\right\}=\left\{1, \ldots, \operatorname{dim}\left(c_{i}\right)\right\} \backslash B_{i} .
\end{aligned}
$$

Consider the set of pairs $(i, r)$ such that $i \in\{1, \ldots, l\}, r \in\left\{1, \ldots, \operatorname{dim}\left(c_{i}\right)\right\}$. We will call these pairs local $\mathfrak{C}$-actions (or local actions if $\mathfrak{C}$ is clear). Let $\sim$ be the equivalence relation on the set of local $\mathfrak{C}$-actions generated by

$$
\left(i, \bar{b}_{i}^{j}\right) \sim\left(i+1, \bar{a}_{i+1}^{j}\right)
$$

for all $i \in\{1, \ldots, l-1\}, j \in\left\{1, \ldots, q_{i}\right\}$.

Definition 4.1 $A \mathfrak{C}$-action (or an action if $\mathfrak{C}$ is clear) is an equivalence class of the relation $\sim$. The set of all $\mathfrak{C}$-actions will be denoted by $T(\mathfrak{C})$. The $\mathfrak{C}$-action represented by $(i, r)$ will be denoted $[i, r]$. 
The following proposition justifies the definition above.

Proposition 4.2 Fix $i \in\{1 \ldots, l-1\}$. Let $\mathbf{x}=\left(x^{1}, \ldots, x^{\operatorname{dim}\left(c_{i}\right)}\right) \in I^{\operatorname{dim}\left(c_{i}\right)}, \mathbf{y}=$ $\left(y^{1}, \ldots, y^{\operatorname{dim}\left(c_{i+1}\right)}\right) \in I^{\operatorname{dim}\left(c_{i+1}\right)}$ be points such that

- $x^{j}=1$ for every $j \in B_{i}$,

- $y^{k}=0$ for every $k \in A_{i+1}$,

- $x^{j}=y^{k}$ whenever $[i, j]=[i+1, k]$.

Then $\left[c_{i} ; \mathbf{x}\right]=\left[c_{i+1} ; \mathbf{y}\right]$.

Proof. Since $x^{j}=1$ for all $j \in B_{i}$ and $y^{k}=0$ for all $k \in A_{i+1}$, we have

$$
\mathbf{x}=\delta_{B_{i}}^{1}\left(x^{\bar{b}_{i}^{1}}, x^{\bar{b}_{i}^{2}}, \ldots, x^{\bar{b}_{i}^{q_{i}}}\right), \quad \mathbf{y}=\delta_{A_{i+1}}^{0}\left(y^{\bar{a}_{i+1}^{1}}, y^{\bar{a}_{i+1}^{2}}, \ldots, y^{\bar{a}_{i+1}^{q_{i}}}\right)
$$

For all $r \in\left\{1, \ldots, q_{i}\right\}$, we have $\left[i, \bar{b}_{i}^{r}\right]=\left[i+1, \bar{a}_{i+1}^{r}\right]$. Therefore,

$$
\begin{aligned}
{\left[c_{i} ; \mathbf{x}\right] } & =\left[d_{B_{i}}^{1}\left(c_{i}\right) ;\left(x^{\bar{b}_{i}^{1}}, x^{\bar{b}_{i}^{2}}, \ldots, x^{\bar{b}_{i}^{q_{i}}}\right)\right] \\
& =\left[d_{A_{i+1}}^{0}\left(c_{i+1}\right) ;\left(y^{\bar{a}_{i+1}^{1}}, y^{\bar{a}_{i+1}^{2}}, \ldots, y^{\bar{a}_{i+1}^{q_{i}}}\right)\right]=\left[c_{i+1}, \mathbf{y}\right]
\end{aligned}
$$

Let us collect some basic properties of $\mathfrak{C}$-actions:

(1) For every $i \in\{1, \ldots, l-1\}$, the sequences of actions

$$
([i, r])_{r \in\left\{1, \ldots, \operatorname{dim}\left(c_{i}\right)\right\} \backslash B_{i}} \quad \text { and } \quad([i+1, s])_{s \in\left\{1, \ldots, \operatorname{dim}\left(c_{i+1}\right)\right\} \backslash A_{i+1}}
$$

are equal.

(2) Every action $p \in T(\mathfrak{C})$ has at most one representative having the form $(i, r)$ for a fixed $i$. If such a representative exists, its second coordinate will be denoted by $r(p, i)$, so that $p=[i, r(p, i)]$. In such a case we will say that the action $p$ is active at the $i$ th stage.

(3) For a given action $p$, the set of stages at which $p$ is active forms a (non-empty) interval, i.e., has the form

$$
\{i: \operatorname{beg}(p) \leq i \leq \operatorname{end}(p)\}
$$

for some integers $1 \leq \operatorname{beg}(p) \leq \operatorname{end}(p) \leq l$. Denote

$$
\begin{aligned}
& T_{i}^{1}(\mathfrak{C})=\{p \in T(\mathfrak{C}) \mid \operatorname{end}(p)<i\} \\
& T_{i}^{*}(\mathfrak{C})=\{p \in T(\mathfrak{C}) \mid \operatorname{beg}(p) \leq i \leq \operatorname{end}(p)\} \\
& T_{i}^{0}(\mathfrak{C})=\{p \in T(\mathfrak{C}) \mid i<\operatorname{beg}(p)\}
\end{aligned}
$$

These are the sets of actions that are finished, active and unstarted, respectively, at the $i$ th stage. 
(4) For every $i$, the following pairs of conditions are equivalent:

$$
\begin{aligned}
& \operatorname{beg}(p)=i \Leftrightarrow p=[i, r] \text { for some } r \in A_{i} \\
& \operatorname{end}(p)=i \Leftrightarrow p=[i, r] \text { for some } r \in B_{i} .
\end{aligned}
$$

(5) We have

$$
\begin{aligned}
\{1, \ldots, l\} & \supsetneq T_{1}^{0}(\mathfrak{C}) \supseteq T_{2}^{0}(\mathfrak{C}) \supseteq \cdots \supseteq T_{k}^{0}(\mathfrak{C})=\emptyset \\
\emptyset & =T_{1}^{1}(\mathfrak{C}) \subseteq T_{2}^{1}(\mathfrak{C}) \subseteq \cdots \subseteq T_{k}^{1}(\mathfrak{C}) \subsetneq\{1, \ldots, l\}
\end{aligned}
$$

\section{Progress functions}

In this section we introduce progress functions, which provide a convenient description of d-paths contained in a given track $\mathfrak{C}$. Fix a bi-pointed $\square$-set $K$, a track $\mathfrak{C}=\left(c_{i}, A_{i}, B_{i}\right)_{i=1}^{l}$ in $K$ and numbers $a<b \in \mathbb{R}$.

Definition 5.1 A progress function of $\mathfrak{C}$ is a sequence $\mathbf{f}=\left(f^{p}\right)_{p \in T(\mathfrak{C})}$ of nondecreasing continuous functions $[a, b] \rightarrow[0,1]$ such that there exist numbers

$$
a=t_{0} \leq t_{1} \leq \cdots \leq t_{k}=b
$$

such that for every $p \in T(\mathfrak{C})$

- $f^{p}(t)=0$ for $t \leq t_{\text {beg }}(p)-1$,

- $f^{p}(t)=1$ for $t \geq t_{\operatorname{end}(p)}$.

Let $\mathrm{PF}_{[a, b]}(\mathfrak{C})$ be the space of progress functions of the track $\mathfrak{C}$, with the compact-open topology.

For every progress function $\mathbf{f} \in \mathrm{PF}_{[a, b]}(\mathfrak{C})$ and an action $p \in T(\mathfrak{C})$ we have $f^{p}(a)=0$ and $f^{p}(b)=1$. Thus, $f^{p}$ can be regarded as a d-path in $\vec{P}_{[a, b]}(\vec{I})_{0}^{1}$, and $\operatorname{PF}_{[a, b]}(\mathfrak{C})$, as a subspace of $\vec{P}_{[a, b]}\left(\vec{I}^{T(\mathfrak{C})}\right)_{\mathbf{0}}^{\mathbf{1}}$. The support of $f^{p}$, defined by

$$
\operatorname{supp}\left(f^{p}\right):=\left\{t \in[a, b] \mid 0<f^{p}(t)<1\right\}
$$

is an open interval. We will denote its endpoints by $a_{\mathbf{f}}^{p}$ and $b_{\mathbf{f}}^{p}$ so that

- $\operatorname{supp}\left(f^{p}\right)=\left(a_{\mathbf{f}}^{p}, b_{\mathbf{f}}^{p}\right)$,

- $f^{p}(t)=0$ for $t \in\left[a, a_{\mathbf{f}}^{p}\right]$,

- $f^{p}(t)=1$ for $t \in\left[b_{\mathbf{f}}^{p}, b\right]$.

For any sequence of numbers $\left(t_{i}\right)$ satisfying Definition 5.1 we have

$$
t_{\operatorname{beg}(p)-1} \leq a_{\mathbf{f}}^{p}<b_{\mathbf{f}}^{p} \leq t_{\mathrm{end}(p)} .
$$

The support of a progress function $\mathbf{f}$ is the set

$$
\operatorname{supp}(\mathbf{f})=\bigcup_{p \in T(C)} \operatorname{supp}\left(f^{p}\right) .
$$


In the remaining part of this section we will describe the relationship between progress functions of $\mathfrak{C}$ and d-paths contained in $\mathfrak{C}$. Let $\mathbf{f}=\left(f^{p}\right)_{p \in T(\mathfrak{C})}$ be a progress function of $\mathfrak{C}$. Our goal is to construct a d-path $\alpha^{\mathbf{f}} \in \vec{P}(K ; \mathfrak{C})_{\mathbf{0}}^{\mathbf{1}}$ that corresponds to $\mathbf{f}$. For every $i \in\{1, \ldots, l\}$ let

$$
a_{i}^{\mathbf{f}}:=\max _{p \in T_{i}^{1}(\mathfrak{C})} b_{\mathbf{f}}^{p}, \quad b_{i}^{\mathbf{f}}:=\min _{p \in T_{i}^{0}(\mathfrak{C})} a_{\mathbf{f}}^{p}
$$

If $T_{i}^{1}(\mathfrak{C})=\emptyset\left(\operatorname{resp} . T_{i}^{0}(\mathfrak{C})=\emptyset\right)$, we take $a_{i}^{\mathbf{f}}=a\left(\right.$ resp. $\left.b_{i}^{\mathbf{f}}=b\right)$. The interval $\left[a_{i}^{\mathbf{f}}, b_{i}^{\mathbf{f}}\right]$ is the maximal one that is disjoint from the supports of functions $f^{p}$ for actions $p$ that are not active at the $i$ th stage. For every $i$ define a d-path $\beta_{i}^{\mathbf{f}} \in \vec{P}_{\left[a_{i}^{\mathbf{f}}, b_{i}^{\mathbf{f}}\right]}\left(\vec{I}^{\operatorname{dim}\left(c_{i}\right)}\right)$ by

$$
\beta_{i}^{\mathbf{f}}(t)=\left(f^{[i, 1]}(t), f^{[i, 2]}(t), \ldots, f^{\left[i, \operatorname{dim}\left(c_{i}\right)\right]}(t)\right)
$$

and let $\alpha_{i}^{\mathbf{f}}=\left[c_{i} ; \beta_{i}^{\mathbf{f}}\right] \in \vec{P}_{\left[a_{i}^{\mathbf{f}}, b_{i}^{\mathbf{f}}\right]}(K)$.

Proposition 5.2 For every progress function $\mathbf{f} \in F_{[a, b]}(\mathfrak{C})$ :

(a) $[a, b]=\bigcup_{i=1}^{l}\left[a_{i}^{\mathbf{f}}, b_{i}^{\mathbf{f}}\right]$,

(b) $\alpha_{i}^{\mathbf{f}}(t)=\alpha_{j}^{\mathbf{f}}(t)$ for all $i, j$ and $t \in\left[a_{i}^{\mathbf{f}}, b_{i}^{\mathbf{f}}\right] \cap\left[a_{j}^{\mathbf{f}}, b_{j}^{\mathbf{f}}\right]$.

Proof By (4.7) we have $a=a_{1}^{\mathbf{f}} \leq a_{2}^{\mathbf{f}} \leq \cdots \leq a_{l}^{\mathbf{f}}$ and $b_{1}^{\mathbf{f}} \leq b_{2}^{\mathbf{f}} \leq \cdots \leq b_{l}^{\mathbf{f}}=b$. Choose a sequence $\left(t_{i}\right)_{i=0}^{l}$ satisfying the condition in Definition 5.1. For every $i \in\{1, \ldots, l-1\}$ we have

$$
\begin{aligned}
a_{i+1}^{\mathbf{f}} & =\max _{p \in T_{i+1}^{1}(\mathfrak{C})} b_{p}^{\mathbf{f}} \stackrel{(5.1)}{\leq} \max _{p \in T_{i+1}^{1}(\mathfrak{C})} t_{\mathrm{end}(p)} \stackrel{(4.5)}{\leq} t_{i} \stackrel{(4.5)}{\leq} \min _{p \in T_{i}^{0}(\mathfrak{C})} t_{\operatorname{beg}(p)-1} \\
& \stackrel{(5.1) \min _{p \in T_{i}^{0}(\mathfrak{C})} a_{p}^{\mathbf{f}}=b_{i}^{\mathbf{f}} .}{ }
\end{aligned}
$$

This implies (a). To prove (b), it is enough to check that $\alpha_{i}^{\mathbf{f}}(t)=\alpha_{i+1}^{\mathbf{f}}(t)$ for every $t \in\left[a_{i+1}^{\mathbf{f}}, b_{i}^{\mathbf{f}}\right]$. For $r \in\left\{1, \ldots, \operatorname{dim}\left(c_{i}\right)\right\}$ we have

$$
\begin{aligned}
r \in B_{i} & \stackrel{(4.6)}{\Rightarrow} \operatorname{end}([i, r])=i \Rightarrow[i, r] \in T_{i+1}^{1}(\mathfrak{C}) \Rightarrow a_{i+1}^{\mathbf{f}} \geq b_{\mathbf{f}}^{[i, r]} \\
& \Rightarrow f^{[i, r]}\left(a_{i+1}^{\mathbf{f}}\right)=1 .
\end{aligned}
$$

Thus, $f^{[i, r]}(t)=1$ for all $r \in B_{i}$. As a consequence,

$$
\left(f^{[i, 1]}(t), \ldots, f^{\left[i, \operatorname{dim}\left(c_{i}\right)\right]}(t)\right)=\delta_{B_{i}}^{1}\left(f^{\left[i, \bar{b}_{i}^{1}\right]}(t), \ldots, f^{\left[i, \bar{b}_{i}^{q_{i}}\right]}(t)\right),
$$

where $\bar{b}_{i}^{j}$ and $q_{i}$ are defined in (4.1). Finally,

$$
\alpha_{i}^{\mathbf{f}}(t)=\left[c_{i} ;\left(f^{[i, 1]}(t), \ldots, f^{\left[i, \operatorname{dim}\left(c_{i}\right)\right]}(t)\right)\right]=\left[c_{i} ; \delta_{B_{i}}^{1}\left(f^{\left[i, \bar{b}_{i}^{1}\right]}(t), \ldots, f^{\left[i, \bar{b}_{i}^{q_{i}}\right]}(t)\right)\right]
$$




$$
=\left[d_{B_{i}}^{1}\left(c_{i}\right) ;\left(f^{\left[i, \bar{b}_{i}^{1}\right]}(t), \ldots, f^{\left[i, \bar{b}_{i}^{q_{i}}\right]}(t)\right)\right]
$$

In a similar way we can show that

$$
\alpha_{i+1}^{\mathbf{f}}(t)=\left[d_{A_{i+1}}^{0}\left(c_{i+1}\right) ;\left(f^{\left[i+1, \bar{a}_{i+1}^{1}\right]}(t), \ldots, f^{\left[i+1, \bar{a}_{i+1}^{q_{i}}\right]}(t)\right)\right] .
$$

Since $d_{A_{i+1}}^{0}\left(c_{i+1}\right)=d_{B_{i}}^{1}\left(c_{i}\right)$ and $\left[i, \bar{b}_{i}^{j}\right]=\left[i+1, \bar{a}_{i+1}^{j}\right]$ for all $j$, the conclusion follows.

Definition 5.3 The d-path associated to a progress function $\mathbf{f} \in \operatorname{PF}_{[a, b]}(\mathfrak{C})$ is the unique d-path $\alpha^{\mathbf{f}} \in \vec{P}_{[a, b]}(K ; \mathfrak{C})$ such that $\alpha(t)=\alpha_{i}^{\mathbf{f}}(t)$ for every $t \in\left[a_{i}^{\mathbf{f}}, b_{i}^{\mathbf{f}}\right]$. Proposition 5.2 guarantees that $\alpha^{\mathbf{f}}$ exists and is determined uniquely.

The construction presented above defines the map

$$
\mathfrak{R}_{\mathfrak{C}}: \mathrm{PF}_{[a, b]}(\mathfrak{C}) \ni \mathbf{f} \mapsto \alpha^{\mathbf{f}} \in \vec{P}_{[a, b]}(K ; \mathfrak{C})
$$

which can be shown to be continuous. We skip a proof of this fact since it is tedious and not necessary for proving the main results of this paper.

Now we will construct a progress function associated to a given d-path $\alpha \in$ $\vec{P}_{[a, b]}(K ; \mathfrak{C})$. Choose a $\mathfrak{C}$-presentation

$$
\alpha={ }^{t_{0}}\left[c_{1} ; \beta_{1}\right] *\left[c_{2} ; \beta_{2}\right] \stackrel{t_{2}}{*} \ldots{ }^{t_{l-1}} *\left[c_{l} ; \beta_{l}\right]^{t_{l}} .
$$

Proposition 5.4 Let $p \in T(\mathfrak{C})$. Then

(a) $\beta_{i}^{r(p, i)}\left(t_{i}\right)=\beta_{i+1}^{r(p, i+1)}\left(t_{i}\right)$ whenever $\operatorname{beg}(p) \leq i<\operatorname{end}(p)$,

(b) $\beta_{i}^{r(p, i)}\left(t_{i-1}\right)=0$ for $i=\operatorname{beg}(p)$,

(c) $\beta_{i}^{r(p, i)}\left(t_{i}\right)=1$ for $i=\operatorname{end}(p)$.

Proof Assume that $\operatorname{beg}(p) \leq i<\operatorname{end}(p)$. Then $r(p, i) \notin B_{i}$ and there exists $j \in$ $\left\{1, \ldots, q_{i}\right\}$ such that $r(p, i)=\bar{b}_{i}^{j}$. Furthermore, $r(p, i+1)=\bar{a}_{i+1}^{j}$. Let $\mathbf{x}_{i} \in \vec{I}^{q_{i}}$ be a point such that $\beta_{i}\left(t_{i}\right)=\delta_{B_{i}}^{1}\left(\mathbf{x}_{i}\right)$ and $\beta_{i+1}\left(t_{i}\right)=\delta_{A_{i+1}}^{0}\left(\mathbf{x}_{i}\right)$. We have

$$
\begin{aligned}
\beta_{i}^{r(p, i)}\left(t_{i}\right) & =\beta_{i}^{\bar{b}_{i}^{j}}\left(t_{i}\right)=\delta_{B_{i}}^{1}\left(\mathbf{x}_{i}\right)^{\bar{b}_{i}^{j}} \stackrel{(2.5)}{=} x_{i}^{j} \stackrel{(2.5)}{=} \delta_{A_{i+1}}^{0}\left(\mathbf{x}_{i}\right)^{\bar{a}_{i+1}^{j}} \\
& =\beta_{i+1}^{\bar{a}_{i+1}^{j}}\left(t_{i}\right)=\beta_{i+1}^{r(p, i+1)}\left(t_{i}\right) .
\end{aligned}
$$

If $i=\operatorname{beg}(p)$, then $r(p, i) \in A_{i}$ and

$$
\beta_{i}^{r(p, i)}\left(t_{i-1}\right)=\delta_{A_{i}}^{0}\left(\mathbf{x}_{i}\right)^{r(p, i)}=0 .
$$

A similar argument shows (c). 
For an arbitrary action $p \in T(\mathfrak{C})$ let us define the function

$$
f_{\alpha}^{p}:[a, b] \ni t \mapsto \begin{cases}0 & \text { for } t \leq t_{\operatorname{beg}(p)-1} \\ \beta_{i}^{r(p, i)}(t) & \text { for } \operatorname{beg}(p) \leq i \leq \operatorname{end}(p) \text { and } t \in\left[t_{i-1}, t_{i}\right] \\ 1 & \text { for } t \geq t_{\operatorname{end}(p)}\end{cases}
$$

and let $\mathbf{f}_{\alpha}=\left(f_{\alpha}^{p}\right)_{p \in T(\mathfrak{C})}$. Proposition 5.4 implies that this definition is valid.

Proposition $5.5 \mathbf{f}_{\alpha}$ is a progress function on $\mathfrak{C}$. Moreover, $\alpha^{\mathbf{f}_{\alpha}}=\alpha$.

Proof. The sequence $\left(t_{i}\right)$ from the presentation (5.6) satisfies the conditions required in Definition 5.1. Notice that $\left[t_{i-1}, t_{i}\right] \subseteq\left[a_{i}^{\mathbf{f}_{\alpha}}, b_{i}^{\mathbf{f}_{\alpha}}\right]$, for all $i \in\{1, \ldots, l\}$. Then, for every $t \in\left[t_{i-1}, t_{i}\right], \alpha_{i}^{\mathbf{f}_{\alpha}}(t)$ is well-defined, and

$$
\begin{aligned}
\alpha^{\mathbf{f}_{\alpha}}(t) & =\alpha_{i}^{\mathbf{f}_{\alpha}}(t)=\left[c_{i} ;\left(f_{\alpha}^{[i, 1]}(t), \ldots, f_{\alpha}^{\left[i, \operatorname{dim}\left(c_{i}\right)\right]}(t)\right)\right] \\
& =\left[c_{i} ;\left(\beta_{i}^{r([i, 1], i)}(t), \ldots, \beta_{i}^{r\left(\left[i, \operatorname{dim}\left(c_{i}\right)\right], i\right)}(t)\right)\right] \\
& =\left[c_{i} ;\left(\beta_{i}^{1}(t), \ldots, \beta_{i}^{\operatorname{dim}\left(c_{i}\right)}(t)\right)\right]=\left[c_{i} ; \beta_{i}(t)\right]=\alpha(t) .
\end{aligned}
$$

The function $\mathbf{f}_{\alpha}$ will be called the progress function of $\alpha \in \vec{P}(K ; \mathfrak{C})$ with the presentation (5.6). Proposition 5.5 implies that the map $\mathfrak{R}_{\mathfrak{C}}$ is surjective. This map is not, in general, a bijection: $\mathbf{f}^{\alpha}$ depends not only on the d-path $\alpha$ but also on the choice of its presentation, as shown in the example below.

Example 5.6 Let $K$ be the $\square$-set having exactly one cube in dimensions $0,1,2$ and no cubes in higher dimensions. Let $e$ be the only 2-dimensional cube and let $\mathfrak{C}=$ $\left(c_{1}=e, A_{1}=\{1,2\}, B_{1}=\{1,2\}\right)$. Let $\alpha \in \vec{P}_{[0,2]}(K)$ be the d-path given by the $\mathfrak{C}$-presentation $\alpha=[e ; \beta]$, where

$$
\beta(t)= \begin{cases}(t, 0) & \text { for } t \in[0,1] \\ (1, t-1) & \text { for } t \in[1,2]\end{cases}
$$

Then $T(\mathfrak{C})=\{[1,1],[1,2]\}$ and the progress function of $\alpha$ is given by

$$
f_{\alpha}^{[1,1]}(t)=\left\{\begin{array}{ll}
t & \text { for } t \in[0,1] \\
1 & \text { for } t \in[1,2] .
\end{array}, \quad f_{\alpha}^{[1,2]}(t)= \begin{cases}0 & \text { for } t \in[0,1] \\
t-1 & \text { for } t \in[1,2]\end{cases}\right.
$$

But $\alpha$ has another $\mathfrak{C}$-presentation, namely $\alpha=\left[e ; \beta^{\prime}\right]$, where

$$
\beta^{\prime}(t)= \begin{cases}(0, t) & \text { for } t \in[0,1] \\ (t-1,1) & \text { for } t \in[1,2]\end{cases}
$$

which gives the progress function with $f_{\alpha}^{[1,1]}$ and $f_{\alpha}^{[1,2]}$ swapped. 
The following observation, which relates the progress function of a d-path with its $L_{1}$-length, plays an important role in the succeeding section. It is an immediate consequence of the definitions.

Proposition 5.7 Fix $\alpha \in \vec{P}_{[a, b]}(K ; \mathfrak{C})$ and its $\mathfrak{C}$-presentation (5.6). For every $t \in[a, b]$ we have

$$
\operatorname{len}\left(\left.\alpha\right|_{[a, t]}\right)=\sum_{p \in T(\mathfrak{C})} f_{\alpha}^{p}(t)
$$

In particular, if $\alpha$ is natural, then $f_{\alpha}^{p}$ is a 1-Lipschitz function for every $p \in T(\mathfrak{C})$.

\section{Tamification theorem}

In this section we will use the results obtained above to prove that the spaces of d-paths and of tame d-paths are homotopy equivalent. The main result is the following:

Theorem 6.1 For every $n \geq 0$ and every $K \in \square$ Set $_{*}^{*}$, all the inclusions in the diagram

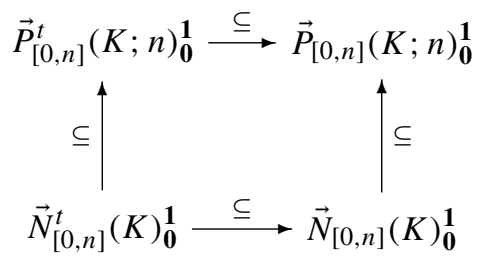

are homotopy equivalences.

For the vertical maps this follows from Raussen (2009). The main idea of the proof of Theorem 6.1 is to construct a functorial self-map of $\vec{P}_{[0, n]}(K ; n)_{\mathbf{0}}^{\mathbf{1}}$ that is homotopic to the identity and maps $\vec{N}_{[0, n]}(K)_{\mathbf{0}}^{\mathbf{1}}$ into $\vec{P}_{[0, n]}^{t}(K ; n)_{\mathbf{0}}^{\mathbf{1}}$.

Any d-map $g: \vec{I} \rightarrow \vec{I}$ that preserves 0 and 1 induces a self-d-map $g^{K}$ of $|K|$ such that

$$
g^{K}\left(\left[c ; h^{1}, \ldots, h^{n}\right]\right)=\left[c ; g\left(h^{1}\right), \ldots, g\left(h^{k}\right)\right]
$$

for every $k \geq 0, c \in K[k]$. Furthermore, a homotopy $g_{s}$ between $g_{0}=\mathrm{id}$ and $g_{1}=g$ induces a homotopy between $g^{K}$ and the identity map on $|K|$. Below we introduce a "path-length-parametrized" analogue of this construction.

Let $R: \overrightarrow{[0, n]} \times \vec{I} \rightarrow \vec{I}$ be an arbitrary d-map such that $R(t, 0)=0$ and $R(t, 1)=1$ for all $t \in[0, n]$. For every $k \geq 0, R$ induces the map

$$
R^{k}: \overrightarrow{[0, n]} \times \vec{I}^{k} \ni\left(t ; h^{1}, \ldots, h^{k}\right) \mapsto\left(R\left(t, h^{1}\right), \ldots, R\left(t, h^{k}\right)\right) \in \vec{I}^{k}
$$


The maps $R^{k}$ are compatible with the face maps, i.e., for every $\varepsilon \in\{0,1\}, k \geq 0$ and $i \in\{1, \ldots, k+1\}$, the diagram

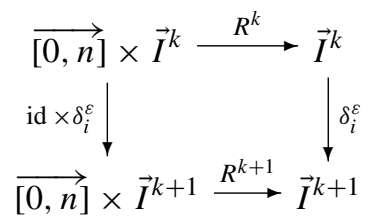

commutes. As a consequence, for every $K \in \square$ Set, the maps $R^{k}$ induce the continuous d-map

$$
R^{K}: \overrightarrow{[0, n]} \times|K| \rightarrow|K|,
$$

such that

$$
R^{K}(t,[c ; \mathbf{x}])=\left[c ; R^{k}(t, \mathbf{x})\right]=\left[c ; R\left(t, x_{1}\right), \ldots, R\left(t, x_{k}\right)\right]
$$

for $c \in K[k], \mathbf{x}=\left(x^{1}, \ldots, x^{k}\right) \in \vec{I}^{k}$.

For $s \in[0,1]$, define the map $R_{s}: \overrightarrow{[0, n]} \times \vec{I} \rightarrow \vec{I}$ by the formula $R_{s}(t, h)=$ $s R(t, h)+(1-s) h$. The collection of maps $R_{s}^{K}: \overrightarrow{[0, n]} \times|K| \rightarrow|K|$ induced by the maps $R_{S}$ is a homotopy between $R^{K}$ and the projection on the second factor.

These maps define, for $0 \leq a \leq b \leq n$, the following self-maps of d-path spaces:

$$
\begin{aligned}
\bar{R}: \vec{P}_{[a, b]}(\vec{I}) & \rightarrow \vec{P}_{[a, b]}(\vec{I}) \\
\bar{R}^{k}: \vec{P}_{[a, b]}\left(\vec{I}^{k}\right) & \rightarrow \vec{P}_{[a, b]}\left(\vec{I}^{k}\right) \\
\bar{R}^{K}: \vec{P}_{[a, b]}(K) & \rightarrow \vec{P}_{[a, b]}(K)
\end{aligned}
$$

such that $\bar{R}(\alpha)(t)=R(t, \alpha(t)), \bar{R}^{k}(\alpha)(t)=R^{k}(t, \alpha(t)), \bar{R}^{K}(\alpha)(t)=R^{K}(t, \alpha(t))$. All these maps are homotopic to the respective identities via the families of maps $\bar{R}_{S}$, $\bar{R}_{s}^{k}$ and $\bar{R}_{s}^{K}$ that are defined in a similar way.

Now assume that $R: \overrightarrow{[0, n]} \times \vec{I} \rightarrow \vec{I}$ satisfies the following conditions:

(a) $R(t, h)=0$ for $h \in\left[0, \frac{1}{4}\right]$,

(b) $R(t, h)=1$ for $h \in\left[\frac{3}{4}, 1\right]$,

(c) For every $h \in[0,1]$, the support of $R(-, h)$,

$$
\operatorname{supp}(R(-, h))=\{t \in[0, n] \mid 0<R(t, h)<1\}
$$

is an interval having length less or equal to $\frac{1}{4 n}$.

Such a function exists; an example is given by the formula (Figs. 2, 3)

$$
R(t, h)=\min \left(1, \max \left(0,\left(4 n t+12 n^{2} h-8 n^{2}\right)\right)\right) .
$$

These assumptions imply the following properties of $\bar{R}$ :

Proposition 6.2 For every $\alpha \in \vec{P}_{[0, n]}(\vec{I})_{0}^{1}$, the support of $\bar{R}(\alpha)$ is an open interval having length less or equal to $\frac{1}{4 n}$. 
Fig. 2 A contour plot of the function $R$ (not up to scale). Values between 0 and 1 are taken only in the narrow stripe
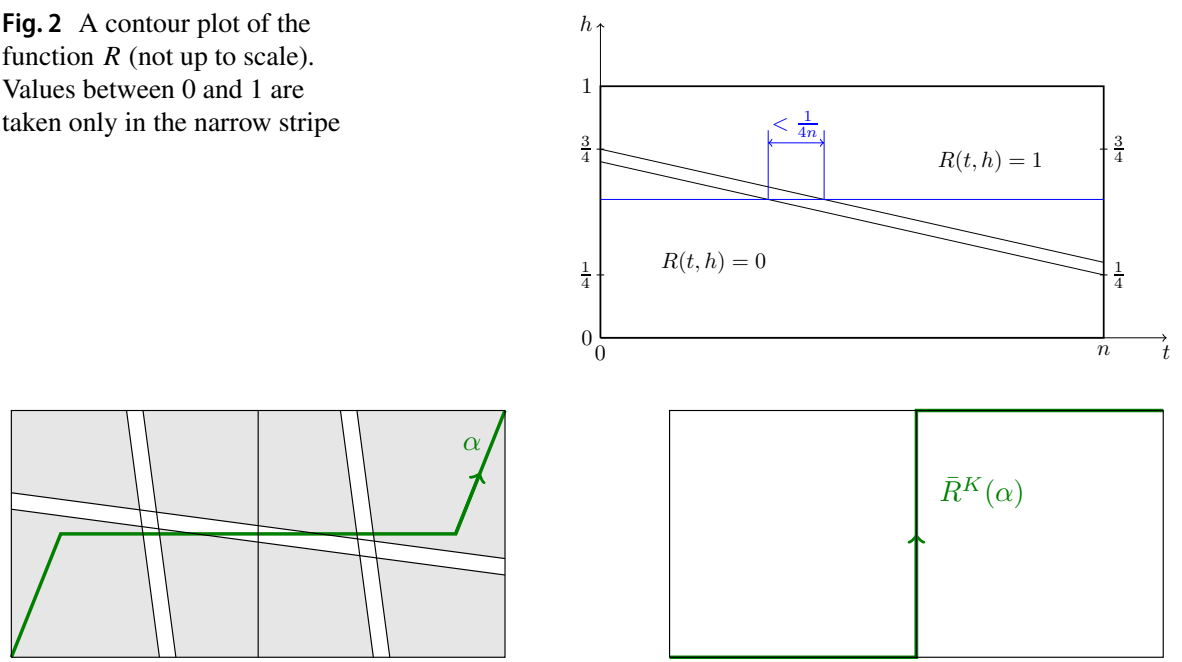

Fig. 3 If a $\square$-set $K$ is simple enough and $\alpha$ is a natural d-path on $K$, then $\bar{R}^{K}(\alpha)=Q \circ \alpha$ for a certain self-map $Q$ of $|K|$. The map $Q$ sends the shaded areas into vertices

Proof Obviously $\bar{R}(\alpha)(0)=R(0, \alpha(0))=0$ and $\bar{R}(\alpha)(n)=R(n, \alpha(n))=1$. Assume that $s<t \in \operatorname{supp}(\bar{R})(\alpha)$, i.e., $0<\bar{R}(\alpha)(s) \leq \bar{R}(\alpha)(t)<1$. Then

$$
0<\bar{R}(\alpha)(s)=R(s, \alpha(s)) \leq R(t, \alpha(s)) \leq R(t, \alpha(t))=\bar{R}(\alpha)(t)<1 .
$$

Hence $s, t \in \operatorname{supp}(R(-, \alpha(s)))$, which implies that $t-s<\frac{1}{4 n}$.

Proposition 6.3 Assume that $\alpha \in \vec{P}_{[0, n]}(\vec{I})_{0}^{1}$ is a 1-Lipschitz function. If $t \in$ $\operatorname{supp}(\bar{R}(\alpha))$, then $\left[t-\frac{1}{4}, t+\frac{1}{4}\right] \subseteq \operatorname{supp}(\alpha)$.

Proof Assume that $\bar{R}(\alpha(t))=R(t, \alpha(t))>0$. By condition (a), we have $\alpha(t)>\frac{1}{4}$, which implies that $\alpha\left(t-\frac{1}{4}\right)>0$. Using condition (b) we obtain that $\alpha(t)<\frac{3}{4}$ and, therefore, $\alpha\left(t+\frac{1}{4}\right)<1$.

Proposition 6.4 If $\alpha \in \vec{N}_{[0, n]}(K)_{\mathbf{0}}^{\mathbf{1}}$, then $\bar{R}^{K}(\alpha)$ is tame.

Proof Denote $\omega=\bar{R}^{K}(\alpha)$. Choose a track $\mathfrak{C}=\left(c_{i}, A_{i}, B_{i}\right)_{i=1}^{l}$ containing $\alpha$, and a $\mathfrak{C}$-presentation

$$
\alpha={ }^{t_{0}}\left[c_{1} ; \beta_{1}\right] * \stackrel{t}{1}^{t_{l-1}} *\left[c_{l} ; \beta_{l}\right]^{t_{l}},
$$

Immediately from the definition follows that

$$
\omega(t)=\left[c_{i} ; R^{\operatorname{dim}\left(c_{i}\right)}\left(t, \beta_{i}(t)\right)\right]
$$

for all $i \in\{1, \ldots, l\}, t \in\left[t_{i-1}, t_{i}\right]$. Thus,

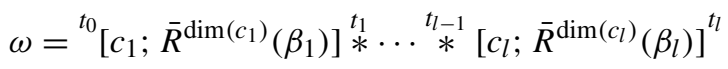


is a $\mathfrak{C}$-presentation of $\omega$. Let $\mathbf{f}_{\alpha}=\left(f_{\alpha}^{p}\right)_{p \in T(\mathfrak{C})}$ and $\mathbf{f}_{\omega}=\left(f_{\omega}^{p}\right)_{p \in T(\mathfrak{C})}$ be the progress functions of $\alpha$ and $\omega$ associated to the presentations (6.6) and (6.8), respectively. Clearly $f_{\omega}^{p}=\bar{R}\left(f_{\alpha}^{p}\right)$ for all $p \in T(\mathfrak{C})$. Recall that

$$
\operatorname{supp}\left(\mathbf{f}_{\omega}\right)=\bigcup_{p \in T(\mathfrak{C})} \operatorname{supp}\left(f_{\omega}^{p}\right)=\bigcup_{p \in T(\mathfrak{C})}\left(a_{\mathbf{f}_{\omega}}^{p}, b_{\mathbf{f}_{\omega}}^{p}\right) .
$$

Let

$$
\operatorname{supp}\left(\mathbf{f}_{\omega}\right)=\left(x_{1}, y_{1}\right) \cup \cdots \cup\left(x_{r}, y_{r}\right) \subseteq(0, n)
$$

be the decomposition into the union of connected components, ordered increasingly. Let $y_{0}=0, x_{r+1}=n$. For every $s \in\{1, \ldots, r\}$, let $A_{s} \subseteq T(\mathfrak{C})$ be the set of actions $p$ such that $\operatorname{supp}\left(f_{\omega}^{p}\right) \subseteq\left(x_{s}, y_{s}\right)$; clearly,

$$
T(\mathfrak{C})=A_{1} \dot{\cup} \ldots \dot{U} A_{r}
$$

For every $s \in\{1, \ldots, r\}$ pick an index $i(s) \in\{1, \ldots, l\}$ such that $\left[t_{i(s)-1}, t_{i(s)}\right] \cap$ $\left(x_{s}, y_{s}\right) \neq \emptyset$. Choose a sequence $\left(z_{s}\right)_{s=0}^{r}$ such that $z_{0}=0, z_{r}=n$ and $y_{s} \leq z_{s} \leq x_{s+1}$ for $s \in\{1, \ldots, r-1\}$. For every $s \in\{0, \ldots, r\}, \omega\left(z_{s}\right)$ is a vertex.

Our goal is to show that

$$
\omega={ }^{z_{0}}\left[c_{i(1)} ; \beta_{i(1)}^{\mathbf{f}^{\omega}}\right] *\left[c_{i(2)} ; \beta_{i(2)}^{\mathbf{f}_{1}}\right]^{z_{2}} \ldots{ }^{z_{l-1}}\left[c_{r} ; \beta_{i(r)}^{\mathbf{f}^{\omega}}\right]^{z_{r}}
$$

is a tame presentation.

By Proposition 6.2, for every $s \in\{1, \ldots, r\}$ the length of the interval $\left(x_{s}, y_{s}\right)$ is less than $\frac{1}{4 n}\left|A_{s}\right| \leq \frac{1}{4}$. Since $f_{\alpha}^{p}$ is a 1-Lipschitz function for every action $p$ (Proposition 5.7), by Proposition 6.3 we have

$$
\left(x_{s}, y_{s}\right) \subseteq \bigcap_{p \in A_{s}} \operatorname{supp}\left(f_{\alpha}^{p}\right)
$$

For every $s \in\{1, \ldots, r\}$ choose $u_{s} \in\left[t_{i(s)-1}, t_{i(s)}\right] \cap\left(x_{s}, y_{s}\right)$.

Fix $s \in\{1, \ldots, r\}$. Assume that $p \in T_{i(s)}^{1}(\mathfrak{C})$. For every $s^{\prime} \geq s$ we have

$$
1=f_{\alpha}^{p}\left(t_{i(s)-1}\right) \leq f_{\alpha}^{p}\left(u_{s}\right) \leq f_{\alpha}^{p}\left(u_{s^{\prime}}\right)
$$

Therefore, $u_{s^{\prime}} \notin \operatorname{supp}\left(f_{\alpha}^{p}\right)$, which implies that $p \notin A_{s^{\prime}}$. As a consequence,

$$
p \in A_{1} \cup \cdots \cup A_{s-1}
$$

and then $b_{\mathbf{f}_{\omega}}^{p} \leq y_{s-1}$. Therefore, for all $s$ we have

$$
a_{i(s)}^{\mathbf{f}_{\omega}}=\max _{p \in T_{i(s)}^{1}(\mathfrak{C})} b_{p}^{\mathbf{f}_{\omega}} \leq y_{s-1}
$$


A similar argument shows that also

$$
b_{i(s)}^{\mathbf{f}_{\omega}}=\min _{p \in T_{i(s)}^{0}(\mathfrak{C})} a_{\mathbf{f}_{\omega}}^{p} \geq x_{s+1} .
$$

Since $\left[z_{s-1}, z_{s}\right] \subseteq\left[y_{s-1}, x_{s+1}\right] \subseteq\left[a_{i(s)}^{\mathbf{f}_{\omega}}, b_{i(s)}^{\mathbf{f}_{\omega}}\right]$, we have

$$
\left.\left.\omega\right|_{\left[z_{s-1}, z_{s}\right]} \stackrel{\text { Prop. } 5.5}{=} \alpha^{\mathbf{f}_{\omega}}\right|_{\left[z_{s-1}, z_{s}\right]}=\left.\alpha_{i(s)}^{\mathbf{f}_{\omega}}\right|_{\left[z_{s-1}, z_{s}\right]}=\left[c_{i(s)} ;\left.\beta_{i(s)}^{\mathbf{f}^{\omega}}\right|_{\left[z_{s-1}, z_{s}\right]}\right],
$$

which shows that $(*)$ is a tame presentation of $\omega$.

Proposition 6.5 For every $s \in[0,1], \bar{R}_{s}^{K}\left(\vec{P}_{[0, n]}^{t}(K)_{\mathbf{0}}^{\mathbf{1}}\right) \subseteq \vec{P}_{[0, n]}^{t}(K)_{\mathbf{0}}^{\mathbf{1}}$.

Proof If $\left.\alpha={ }^{t_{0}}\left[c_{1} ; \beta_{1}\right] *{ }^{t_{1}} *{ }^{t_{l}-1} *{ }^{t_{l}} ; \beta_{l}\right]^{t_{l}}$ is a tame presentation, then also

$$
\left.\bar{R}_{s}^{K}(\alpha)={ }^{t_{0}}\left[c_{1} ; \bar{R}_{s}^{\operatorname{dim}\left(c_{1}\right)}\left(\beta_{1}\right)\right]\right]^{t_{1}} \cdots{ }^{t_{l-1}} *\left[c_{r} ; \bar{R}_{s}^{\operatorname{dim}\left(c_{r}\right)}\left(\beta_{r}\right)\right]^{t_{l}}
$$

is a tame presentation, since $\bar{R}_{s}^{\operatorname{dim}\left(c_{i}\right)}\left(\beta_{i}\right)\left(t_{i-1}\right)=\mathbf{0}$ and $\bar{R}_{s}^{\operatorname{dim}\left(c_{i}\right)}\left(\beta_{i}\right)\left(t_{i}\right)=\mathbf{1}$ for all $i$.

Proof of Theorem 6.1 By Proposition 6.4, the diagram (6.1) can be completed to the diagram

$$
\begin{gathered}
\vec{P}_{[0, n]}^{t}(K ; n)_{\mathbf{0}}^{\mathbf{1}} \stackrel{\subseteq}{\longrightarrow} \vec{P}_{[0, n]}(K ; n)_{\mathbf{0}}^{\mathbf{1}} \\
\subseteq \simeq \simeq \subseteq \\
\vec{N}_{[0, n]}^{t}(K)_{\mathbf{0}}^{\mathbf{1}} \stackrel{\bar{R}^{K}}{\longrightarrow} \vec{N}_{[0, n]}(K)_{\mathbf{0}}^{\mathbf{1}}
\end{gathered}
$$

Since $\bar{R}^{K}: \vec{P}_{[0, n]}(K ; n)_{\mathbf{0}}^{\mathbf{1}} \rightarrow \vec{P}_{[0, n]}(K ; n)_{\mathbf{0}}^{\mathbf{1}}$ is homotopic to the identity via the maps $\bar{R}_{s}^{K}$, the right-hand triangle of the diagram commutes up to homotopy. For similar reasons, Proposition 6.5 implies that also the left-hand triangle commutes up to homotopy. Both vertical inclusions are homotopy equivalences: their homotopy inverses are the naturalization maps (see Sect. 2.8), since the a naturalization of a tame d-path is tame. Now an easy diagram-chasing argument shows that all the maps in the diagram are homotopy equivalences.

Let $\operatorname{Tam}_{n}^{K}: \vec{P}_{[0, n]}(K ; n)_{\mathbf{0}}^{\mathbf{1}} \rightarrow \vec{N}_{[0, n]}^{t}(K)_{\mathbf{0}}^{\mathbf{1}}$ be the composition

$$
\vec{P}_{[0, n]}(K ; n)_{\mathbf{0}}^{\mathbf{1}} \stackrel{\operatorname{nat}_{n}^{K}}{\longrightarrow} \vec{N}_{[0, n]}(K)_{\mathbf{0}}^{\mathbf{1}} \stackrel{\bar{R}^{K}}{\longrightarrow} \vec{P}_{[0, n]}^{t}(K ; n)_{\mathbf{0}}^{\mathbf{1}} \stackrel{\text { nat }_{n}^{K}}{\longrightarrow} \vec{N}_{[0, n]}^{t}(K)_{\mathbf{0}}^{\mathbf{1}}
$$

The following is an immediate consequence of Theorem 6.1: 
Corollary 6.6 For every $n \geq 0$ and every bi-pointed $\square$-set $K \in \square$ Set $_{*}^{*}$, the map $\operatorname{Tam}_{n}^{K}$ is a homotopy inverse of the inclusion $i_{n}^{K}: \vec{N}_{[0, n]}^{t}(K)_{\mathbf{0}}^{\mathbf{1}} \subseteq \vec{P}_{[0, n]}(K ; n)_{\mathbf{0}}^{\mathbf{1}}$. Furthermore, the maps $\operatorname{Tam}_{n}^{K}$ are functorial with respect to $K$, i.e., they define the natural transformation $\vec{P}_{[0, n]}(-; n)_{\mathbf{0}}^{\mathbf{1}} \Rightarrow \vec{N}_{[0, n]}^{t}(-)_{\mathbf{0}}^{\mathbf{1}}$ of functors $\square$ Set $_{*}^{*} \rightarrow$ Top.

\section{Cube chains}

In this section we define the cube chain category $\operatorname{Ch}(K)$ of a bi-pointed $\square$-set $K$, and formulate the main results of the paper.

Definition 7.1 Let $\left(K, \mathbf{0}_{K}, \mathbf{1}_{K}\right),\left(L, \mathbf{0}_{L}, \mathbf{1}_{L}\right)$ be bi-pointed $\square$-sets. The sequential wedge, or the wedge of $K$ and $L$ is a bi-pointed $\square$-set $\left(K \vee L, \mathbf{0}_{K \vee L}, \mathbf{1}_{K \vee L}\right)$ such that

- $(K \vee L)[n]=K[n] \bigsqcup L[n]$ for $n>0$,

- $(K \vee L)[0]=(K[0] \bigsqcup L[0]) / \mathbf{1}_{K} \sim \mathbf{0}_{L}$,

- the face maps of $K \vee L$ are the disjoints unions of the face maps of $K$ and $L$,

- $\mathbf{0}_{K \vee L}=\mathbf{0}_{K}, \mathbf{1}_{K \vee L}=\mathbf{1}_{L}$.

Remark The sequential wedge operation is associative but not commutative. Similarly, one can define the "parallel" wedge of $K$ and $L$ by identifying $\mathbf{0}_{K}$ with $\mathbf{0}_{L}$ and $\mathbf{1}_{K}$ with $\mathbf{1}_{L}$ in $K \bigsqcup L$. This will not be needed here; in this paper we use only sequential wedges.

Let $\operatorname{Seq}(n)$ be the set of sequences of positive integers $\mathbf{n}=\left(n_{1}, \ldots, n_{l}\right)$ such that $n_{1}+\cdots+n_{l}=n$. For $\mathbf{n} \in \operatorname{Seq}(n)$,

- $|\mathbf{n}|=n$ is the length of $\mathbf{n}$,

- $l(\mathbf{n})=l$ is the number of elements of $\mathbf{n}$,

- $t_{i}^{\mathbf{n}}=\sum_{j=1}^{i} n_{j}$ for $i \in\{0, \ldots, l(\mathbf{n})\}$ is the $i$ th vertex of $\mathbf{n}$,

- $\operatorname{Vert}(\mathbf{n})=\left\{t_{i}^{\mathbf{n}}\right\}_{i=0}^{l(\mathbf{n})}$ is the set of vertices of $\mathbf{n}$.

Definition 7.2 Let $\mathbf{n} \in \operatorname{Seq}(n)$. The wedge $\mathbf{n}$-cube is the bi-pointed $\square$-set

$$
\square^{\vee \mathbf{n}}:=\square^{n_{1}} \vee \square^{n_{2}} \vee \cdots \vee \square^{n_{l}},
$$

where $\square^{n_{i}}$ is regarded as a bi-pointed $\square$-set by taking $\mathbf{0}=(0, \ldots, 0), \mathbf{1}=(1, \ldots, 1)$. The geometric realization of $\square^{\vee \mathbf{n}}$ will be denoted by $\vec{I}^{\vee \mathbf{n}}$. For $i \in\{0, \ldots, l(\mathbf{n})\}$,

$$
v_{i}^{\mathbf{n}}=\mathbf{1}_{\square}^{n_{i}}=\mathbf{0}_{\square^{n_{i+1}}} \in \square^{\vee \mathbf{n}_{[0]}}
$$

is the $i$ th vertex of $\square^{\vee \mathbf{n}}$.

Let $\square \mathbf{C h}_{n}$ be the full subcategory of $\square$ Set $_{*}^{*}$ with objects $\square^{\vee \mathbf{n}}$ for $\mathbf{n} \in \operatorname{Seq}(n)$. We will introduce a notation for some morphisms of $\square \mathbf{C h}_{n}$. Let $u_{n} \in \square^{n}[n]$ be the unique top dimension cube. For a partition

$$
\left\{1, \ldots, m_{1}+m_{2}\right\}=A \dot{\cup} B, \quad|A|=m_{2}>0, \quad|B|=m_{1}>0,
$$


let $\varphi_{A, B}: \square^{m_{1}} \vee \square^{m_{2}} \rightarrow \square^{m_{1}+m_{2}}$ be the unique bi-pointed $\square$-map such that $\varphi_{A, B}\left(u_{m_{1}}\right)=d_{B}^{0}\left(u_{m_{1}+m_{2}}\right)$ and $\varphi_{A, B}\left(u_{m_{2}}\right)=d_{A}^{1}\left(u_{m_{1}+m_{2}}\right)$. For $\mathbf{n} \in \operatorname{Seq}(n)$, $i \in\{1, \ldots, l(\mathbf{n})\}$ and $A \dot{\cup} B=\left\{1, \ldots, n_{i}\right\}$ let

$$
\begin{aligned}
& \delta_{i, A, B}: \square^{n_{1}} \vee \cdots \vee \square^{n_{i-1}} \vee \square^{|A|} \vee \square^{|B|} \vee \square^{n_{i+1}} \vee \cdots \square^{n_{l}} \\
& \stackrel{\operatorname{id}_{\square^{n_{1}}} \vee \cdots \vee \operatorname{id}_{\square^{n_{i-1}}} \vee \varphi_{A, B} \vee \mathrm{id}_{\square^{n_{i+1}}} \vee \cdots \vee \mathrm{vid}_{\square^{n_{l}}}}{\longrightarrow} \square^{n_{1}} \\
& \vee \cdots \vee \square^{n_{i-1}} \vee \square^{n_{i}} \vee \square^{n_{i+1}} \vee \cdots \square^{n_{l}}=\square^{\vee \mathbf{n}} \text {. }
\end{aligned}
$$

Fix a bi-pointed $\square$-set $K \in \square$ Set $_{*}^{*}$.

Definition 7.3 A cube chain in $K$ (of length $n$ ) is a bi-pointed $\square$-map c : $\square^{\vee \mathbf{n}^{\mathbf{c}}} \rightarrow K$ for some $\mathbf{n}^{\mathbf{c}} \in \operatorname{Seq}(n)$. The multi-index $\mathbf{n}=\mathbf{n}^{\mathbf{c}}$ will be called the type of $\mathbf{c}$. For short we denote $l(\mathbf{c})=l\left(\mathbf{n}^{\mathbf{c}}\right), t_{i}^{\mathbf{c}}=t_{i}^{\mathbf{n}_{\mathbf{c}}}$.

There is a 1-1 correspondence between cube chains in $K$ and sequences of cubes $\left(c_{1}, \ldots, c_{l}\right)$ such that $d^{0}\left(c_{1}\right)=\mathbf{0}_{K}, d^{1}\left(c_{l}\right)=\mathbf{1}_{K}$ and $d^{1}\left(c_{i}\right)=d^{0}\left(c_{i+1}\right)$ for all $i \in\{1, \ldots, l-1\}$. Indeed, a cube chain $\mathbf{c}: \square^{\vee \mathbf{n}} \rightarrow K$ determines the sequence $\left(\mathbf{c}\left(u_{\operatorname{dim}\left(c_{i}\right)}\right)\right)_{i=1}^{l}$, and a sequence $\left(c_{i}\right)_{i=1}^{l}$ determines the unique $\square$-map

$$
\mathbf{c}: \square^{\operatorname{dim}\left(c_{1}\right)} \vee \cdots \vee \square^{\operatorname{dim}\left(c_{l}\right)} \rightarrow K
$$

such that $\mathbf{c}\left(u_{\operatorname{dim}\left(c_{i}\right)}\right)=c_{i}$. This shows that the notion of a cube chain defined here coincides with the definition introduced in Ziemiański (2017). Below, we will identify cube chains $\mathbf{c}$ and the corresponding sequences $\left(c_{1}, \ldots, c_{l}\right)$ satisfying the conditions above.

Definition 7.4 The length $n$ cube chain category $\mathrm{Ch}(K ; n)$ of $K$ is the slice category $\square \mathbf{C h}_{n} \downarrow K$. In other words, objects of $\mathrm{Ch}(K ; n)$ are cube chains of length $n$, and morphisms from $\mathbf{a}$ to $\mathbf{b}$ are commutative diagrams

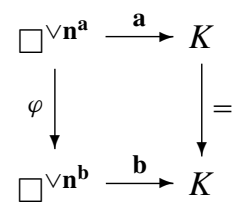

in $\square$ Set $_{*}^{*}$. The full cube chain category of $K$ is $\mathrm{Ch}(K):=\coprod_{n \geq 0} \mathrm{Ch}(K ; n)$.

For $\mathbf{c} \in \operatorname{Ch}(K ; n), i \in\left\{1, \ldots, l\left(\mathbf{n}^{\mathbf{c}}\right)\right\}$ and $A \dot{\cup} B=\left\{1, \ldots, n_{i}^{\mathbf{c}}\right\}$ denote $d_{i, A, B}(\mathbf{c})=$ $\mathbf{c} \circ \delta_{i, A, B}$. The morphism $d_{i, A, B}(\mathbf{c}) \rightarrow \mathbf{c}$ of $\mathrm{Ch}(K ; n)$ that is given by the composition with $\delta_{i, A, B}$ will be also denoted by $\delta_{i, A, B}$.

There is a forgetful functor dom : $\mathrm{Ch}(K ; n) \rightarrow \square$ Set $_{*}^{*}$ that assigns to every cube chain $\mathbf{c}: \square^{\vee \mathbf{n}^{\mathbf{c}}} \rightarrow K$ its domain $\square^{\vee \mathbf{n}^{\mathbf{c}}}$. This is equipped with the natural transformation dom $\rightarrow$ const $_{K}$, which is induced by the chains themselves. For every $n \geq 0$, this transformation induces the map

$$
F_{n}^{K}: \underset{\mathbf{a} \in \operatorname{Ch}_{n}(K ; n)_{\mathbf{0}}^{\mathbf{1}}}{\operatorname{colim}} \vec{N}_{[0, n]}\left(\square^{\vee \mathbf{n}^{\mathbf{a}}}\right)_{\mathbf{0}}^{\mathbf{1}} \rightarrow \vec{N}_{[0, n]}^{t}(K)_{\mathbf{0}}^{\mathbf{1}}
$$


into the space of tame d-paths, since all d-paths in $\vec{N}_{[0, n]}\left(\square^{\vee \mathbf{n}^{\mathbf{a}}}\right)_{\mathbf{0}}^{\mathbf{1}}$ are tame; see the discussion in the beginning of the next section for details.

Consider the sequence of maps

$$
|\mathrm{Ch}(K ; n)| \stackrel{A_{n}^{K}}{\longleftarrow} \underset{\mathbf{c} \in \operatorname{Ch}(K ; n)}{\operatorname{hocolim}} \vec{N}\left(\square^{\vee \mathbf{n}^{\mathbf{c}}}\right)_{\mathbf{0}}^{\mathbf{1}} \stackrel{Q_{n}^{K}}{\longrightarrow} \underset{\mathbf{c} \in \operatorname{Ch}(K ; n)}{\operatorname{colim}} \vec{N}\left(\square^{\vee \mathbf{n}^{\mathbf{c}}}\right)_{\mathbf{0}}^{\mathbf{1}} \stackrel{F_{n}^{K}}{\longrightarrow} \vec{N}_{[0, n]}^{t}(K)_{\mathbf{0}}^{\mathbf{1}},
$$

where $A_{n}^{K}$ is the composition

$$
\underset{\mathbf{c} \in \operatorname{Ch}(K ; n)}{\operatorname{hocolim}} \vec{N}\left(\square^{\vee \mathbf{n}^{\mathbf{c}}}\right)_{\mathbf{0}}^{\mathbf{1}} \rightarrow \underset{\mathbf{c} \in \operatorname{Ch}(K ; n)}{\operatorname{hocolim}}\{*\} \cong|\mathrm{Ch}(K ; n)|,
$$

and $Q_{n}^{K}$ is the natural map from the homotopy colimit to the colimit of the functor $\vec{N}\left(\square^{\vee n^{(-)}}\right)_{\mathbf{0}}^{\mathbf{1}}$.

Theorem 7.5 For every $K \in \square$ Set $_{*}^{*}$, all the maps in the sequence (7.5) are weak homotopy equivalences.

Proof It follows from Ziemiański (2017, Proposition 6.2.(1)) that the space $\vec{N}_{[0, n]}\left(\square^{\vee \mathbf{n}}\right)_{\mathbf{0}}^{\mathbf{1}}$ is contractible for every $\mathbf{n} \in \operatorname{Seq}(n)$. As a consequence, the map $A_{n}^{K}$ is a weak homotopy equivalence by Dugger (2008, Proposition 4.7). The maps $Q_{n}^{K}$ and $F_{n}^{K}$ are weak homotopy equivalences by Propositions 10.4 and 9.7, respectively.

As an immediate consequence, we obtain the main result of this paper.

Theorem 7.6 The functors

$$
\square \mathbf{S e t}_{*}^{*} \ni(K, \mathbf{0}, \mathbf{1}) \mapsto \vec{P}(K)_{\mathbf{0}}^{\mathbf{1}} \in \mathbf{h T o p}
$$

and

$$
\square \mathbf{S e t}_{*}^{*} \ni(K, \mathbf{0}, \mathbf{1}) \mapsto|\mathrm{Ch}(K)| \in \mathbf{h T o p}
$$

are naturally equivalent. In particular, for every bi-pointed $\square$-set $K$, the spaces $\vec{P}(K)_{\mathbf{0}}^{\mathbf{1}}$ and $|\mathrm{Ch}(K)|$ are homotopy equivalent.

Proof For every $K \in \square$ Set $_{*}^{*}$, there is a sequence of maps

$$
|\operatorname{Ch}(K)|=\coprod_{n \geq 0}|\operatorname{Ch}(K ; n)| \underset{\text { Theorem 7.5 }}{\stackrel{\simeq}{\longrightarrow}} \coprod_{n \geq 0} \vec{N}_{[0, n]}^{t}(K ; n)_{\mathbf{0}}^{\mathbf{1}} \underset{\text { Theorem 6.1 }}{\stackrel{\subseteq}{\longrightarrow}} \coprod_{n \geq 0} \vec{P}(K ; n)_{\mathbf{0}}^{\mathbf{1}}=\overrightarrow{\overline{(2.9)}} \vec{P}(K)_{\mathbf{0}}^{\mathbf{1}}
$$

which are all functorial weak homotopy equivalences. 


\section{Natural tame presentations}

In this section we study properties of presentations of natural tame paths on an arbitrary bi-pointed $\square$-set $K$. Fix an integer $n \geq 0$, which will be the length of all d-paths considered here.

If

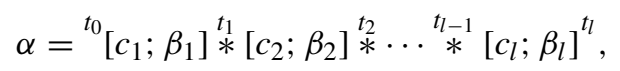

is a tame presentation of a tame natural d-path $\alpha \in \vec{N}_{[0, n]}^{t}(K)_{\mathbf{0}}^{\mathbf{1}}$, then $\mathbf{c}=\left(c_{1}, \ldots, c_{l}\right)$ is a cube chain in $K$, and

$$
\beta=\beta_{1} * \beta_{2} * \cdots * \beta_{l} \in \vec{N}_{[0, n]}\left(\vec{I}^{\vee \mathbf{n}^{\mathbf{c}}}\right)_{\mathbf{0}}^{\mathbf{1}}
$$

is a natural d-path such that $\alpha=[\mathbf{c} ; \beta]=|\mathbf{c}| \circ \beta$. For every pair $(\mathbf{c}, \beta)$ such that c $\in \mathrm{Ch}(K ; n)$ and $\beta \in \vec{N}_{[0, n]}(K)_{\mathbf{0}}^{\mathbf{1}}$ we can associate the presentation (8.1) when we take $\beta_{i}=\left.\beta\right|_{\left[t_{i-1}^{\mathbf{c}}, t_{i}^{\mathbf{c}}\right]}$, regarded as a natural d-path in $\vec{I}_{i}^{n_{i}^{\mathbf{c}}} \subseteq \vec{I}^{\vee \mathbf{n}^{\mathbf{c}}}$. This is construction is correct: since $\beta\left(t_{i}^{\mathbf{c}}\right)=v_{i}^{\mathbf{n}^{\mathbf{c}}}$, the whole segment $\beta_{i}$ lies in the $i$ th cube of the wedge cube $\vec{I}^{\vee \mathbf{n}^{\mathbf{c}}}$. This identifies tame presentations of natural paths having length $n$ with elements of $\bigsqcup_{\mathbf{c} \in \operatorname{Ch}(K ; n)} \vec{N}_{[0, n]}\left(\vec{I}^{\vee \mathbf{n}^{\mathbf{c}}}\right)_{\mathbf{0}}^{\mathbf{1}}$ that are mapped into $\alpha$ by the composition

$$
\coprod_{\mathbf{c} \in \operatorname{Ch}(K ; n)} \vec{N}_{[0, n]}\left(\vec{I}^{\vee \mathbf{n}^{\mathbf{c}}}\right)_{\mathbf{0}}^{\mathbf{1}} \rightarrow \underset{\mathbf{c} \in \operatorname{Ch}(K ; n)}{\operatorname{colim}} \vec{N}_{[0, n]}\left(\vec{I}^{\vee \mathbf{n}^{\mathbf{c}}}\right)_{\mathbf{0}}^{\mathbf{1}} \stackrel{F_{K}^{n}}{\longrightarrow} \vec{N}_{[0, n]}^{t}(K)_{\mathbf{0}}^{\mathbf{1}}
$$

Thus, elements of $\coprod_{\mathbf{c} \in \operatorname{Ch}(K ; n)} \vec{N}_{[0, n]}\left(\vec{I}^{\mathbf{n}^{\mathbf{c}}}\right)_{\mathbf{0}}^{\mathbf{1}}$ will be also called natural tame presentations, or nt-presentations for short.

For any d-path $\alpha \in \vec{P}_{[a, b]}(K)$ denote

$$
\operatorname{Vert}(\alpha)=\alpha^{-1}\left(\left|K_{(0)}\right|\right)=\{t \in[a, b] \mid \alpha(t) \text { is a vertex }\}
$$

Note that if $\alpha \in \vec{N}_{[0, n]}(K)_{\mathbf{0}}^{\mathbf{1}}$, then $\operatorname{Vert}(\alpha) \subseteq\{0,1, \ldots, n\}$. If additionally $\alpha$ admits a natural tame presentation $\alpha=[\mathbf{c} ; \beta]$, then $\operatorname{Vert}\left(\mathbf{n}^{\mathbf{c}}\right) \subseteq \operatorname{Vert}(\alpha)$.

Definition 8.1 A natural tame presentation $\alpha=[\mathbf{c} ; \beta]$ is

(a) minimal if $\operatorname{Vert}(\alpha)=\operatorname{Vert}\left(\mathbf{n}^{\mathbf{c}}\right)$,

(b) regular if for every $i \in\{1, \ldots, l(\mathbf{c})\}$ there exists $t \in\left[t_{i-1}^{\mathbf{c}}, t_{i}^{\mathbf{c}}\right]$ such that $\beta_{i}(t) \in$ $(0,1)^{n_{i}^{\mathbf{c}}}$

(c) equivalent to a natural tame presentation $\alpha=\left[\mathbf{c}^{\prime} ; \beta^{\prime}\right]$ if both $(\mathbf{c}, \beta)$ and $\left(\mathbf{c}^{\prime}, \beta^{\prime}\right)$ represent the same element in $\operatorname{colim}_{\mathbf{c} \in \operatorname{Ch}(K ; n)} \vec{N}_{[0, n]}\left(\vec{I}^{\vee \mathbf{n}^{\mathbf{c}}}\right)_{\mathbf{0}}^{\mathbf{1}}$.

Equivalently, $\alpha=[\mathbf{c} ; \beta]$ is not regular if $\beta_{i} \in \vec{N}_{\left[t_{i-1}^{\mathbf{c}}, t_{i}^{\mathbf{c}}\right]}\left(\partial \vec{I}_{i}^{\mathbf{c}}\right)$ for some $i \in$ $\{1, \ldots, l(\mathbf{c})\}$.

The set of equivalence classes of nt-presentations of $\alpha$ is just $\left(F_{K}^{n}\right)^{-1}(\alpha)$. The example below shows that there may exist non-equivalent nt-presentations of a given natural tame path. 
Example 8.2 Let $K=\square^{3} \cup_{\partial} \square^{3} \square^{3}$ be the union of two standard 3-cubes, glued along their boundaries. Denote the 3-cubes of $K$ by $c$ and $c^{\prime}$ and choose a d-path $\alpha \in \vec{N}\left(\partial \vec{I}^{3}\right)_{\mathbf{0}}^{\mathbf{1}}$ that is not tame. But $\alpha$ regarded as a d-path in $K$ is tame; it has two different tame presentations: $[c ; \alpha]$ and $\left[c^{\prime} ; \alpha\right]$, which are not equivalent. The two presentations are not regular.

Proposition 8.3 Assume that $\alpha=[\mathbf{c} ; \beta]$ is an nt-presentation. Assume that $k \in$ $\operatorname{Vert}(\alpha) \backslash \operatorname{Vert}\left(\mathbf{n}^{\mathbf{c}}\right)$. Then there exists an nt-presentation $\alpha=\left[\mathbf{c}^{\prime} ; \beta^{\prime}\right]$ that is equivalent to $[\mathbf{c} ; \beta]$ such that $\operatorname{Vert}\left(\mathbf{n}^{\mathbf{c}^{\prime}}\right)=\operatorname{Vert}\left(\mathbf{n}^{\mathbf{c}}\right) \cup\{k\}$.

Proof Let $i \in\{1, \ldots, l(\mathbf{c})\}$ be an integer such that $t_{i-1}^{\mathbf{c}}<k<t_{i}^{\mathbf{c}}$. We have $\beta_{i}(k) \in$ $\{0,1\}^{n_{i}^{\mathrm{c}}}$; denote

$$
A=\left\{j \in\left\{1, \ldots, n_{i}^{\mathbf{c}}\right\} \mid \beta_{i}^{j}(k)=1\right\}, \quad B=\left\{j \in\left\{1, \ldots, n_{i}^{\mathbf{c}}\right\} \mid \beta_{i}^{j}(k)=0\right\} .
$$

Obviously $\left.\beta_{i}^{j}\right|_{\left[t_{i-1}^{\mathbf{c}}, k\right]} \equiv 0$ for all $j \in B$ and $\left.\beta_{i}^{j}\right|_{\left[k, t_{i}^{\mathbf{c}}\right]} \equiv 1$ for all $j \in A$. Therefore, there exist unique paths $\gamma \in \vec{N}_{\left[t_{i-1}^{\mathbf{c}}, k\right]}\left(\vec{I}^{|A|}\right)_{\mathbf{0}}^{\mathbf{1}}, \gamma^{\prime} \in \vec{N}_{\left[k, t_{i}^{\mathbf{c}}\right]}\left(\overrightarrow{I^{|B|}}\right)_{\mathbf{0}}^{\mathbf{1}}$ such that $\left.\beta_{i}\right|_{\left[t_{i-1}^{\mathbf{c}}, k\right]}=$ $\delta_{B}^{0}(\gamma),\left.\beta_{i}\right|_{\left[k, t_{i}^{\mathbf{c}}\right]}=\delta_{A}^{1}\left(\gamma^{\prime}\right)$. As a consequence,

$$
\left[c_{i} ; \beta_{i}\right]=\left[c_{i} ; \delta_{B}^{0}(\gamma)\right] *\left[c_{i} ; \delta_{A}^{1}\left(\gamma^{\prime}\right)\right]=\left[d_{B}^{0}\left(c_{i}\right) ; \gamma\right] *\left[d_{A}^{1}\left(c_{i}\right) ; \gamma^{\prime}\right]
$$

Let

$$
\begin{aligned}
& \mathbf{c}^{\prime}=\left(c_{1}, \ldots, c_{i-1}, d_{B}^{0}\left(c_{i}\right), d_{A}^{1}\left(c_{i}\right), c_{i+1}, \ldots, c_{l(\mathbf{c})}\right) \in \mathrm{Ch}(K ; n), \\
& \beta^{\prime}=\beta_{1} * \cdots * \beta_{i-1} * \gamma * \gamma^{\prime} * \beta_{i+1} * \cdots * \beta_{l(\mathbf{c})} \in \vec{N}_{[0, n]}\left(\vec{I}^{\vee \mathbf{n}^{\mathbf{c}^{\prime}}}\right)_{\mathbf{0}}^{\mathbf{1}}
\end{aligned}
$$

We have $d_{i, A, B}(\mathbf{c})=\mathbf{c}^{\prime}$. The map

$$
\vec{N}_{[0, n]}\left(\delta_{i, A, B}\right)_{\mathbf{0}}^{\mathbf{1}}: \vec{N}_{[0, n]}\left(\vec{I}^{\vee \mathbf{n}^{\mathbf{c}^{\prime}}}\right) \rightarrow \vec{N}_{[0, n]}\left(\vec{I}^{\vee \mathbf{n}^{\mathbf{c}}}\right)
$$

induced by the morphism $\delta_{i, A, B}: \mathbf{c}^{\prime} \rightarrow \mathbf{c}$ sends $\beta$ into $\beta^{\prime}$. As a consequence, the presentations $[\mathbf{c} ; \beta]$ and $\left[\mathbf{c}^{\prime}, \beta^{\prime}\right]$ are equivalent. The condition $\operatorname{Vert}\left(\mathbf{n}^{\mathbf{c}^{\prime}}\right)=\operatorname{Vert}\left(\mathbf{n}^{\mathbf{c}}\right) \cup$ $\{k\}$ follows immediately from the definition of $\mathbf{c}^{\prime}$.

Proposition 8.4 Every natural tame presentation is equivalent to a minimal natural tame presentation.

Proof This follows by induction from Proposition 8.3.

Proposition 8.5 Every regular nt-presentation is minimal.

Proof Assume that $[\mathbf{c}, \beta]$ is a regular nt-presentation. Then, for every $i \in\{1, \ldots, l(\mathbf{c})\}$ there exists $t_{i-1}^{\mathbf{n}^{\mathbf{c}}}<t<t_{i}^{\mathbf{n}^{\mathbf{c}}}$ such that $\beta_{i}(t) \in(0,1)^{\operatorname{dim}\left(c_{i}\right)}$, which implies that $\operatorname{Vert}\left(\beta_{i}\right)=\left\{t_{i-1}^{\mathbf{n}^{\mathbf{c}}}, t_{i}^{\mathbf{n}^{\mathbf{c}}}\right\}$. Since $\operatorname{Vert}(\alpha)=\bigcup_{i} \operatorname{Vert}\left(\beta_{i}\right)$, the presentation $[\mathbf{c} ; \beta]$ is minimal. 
A d-path $\alpha \in \vec{N}_{[0, n]}^{t}(K)_{\mathbf{0}}^{\mathbf{1}}$ is regular if it admits a regular presentation. To prove that all nt-presentations of regular d-paths are equivalent, we need the following.

Lemma 8.6 Let $\alpha, \beta \in \vec{P}_{[a, b]}\left(\vec{I}^{m}\right)$ be d-paths such that:

(a) $\alpha(a)=\beta(a)=\mathbf{0}$,

(b) $\alpha(b)=\beta(b) \notin \partial \overrightarrow{\vec{I}^{n}}$, i.e., $0<\alpha^{j}(b)=\beta^{j}(b)<1$ for all $j$,

(c) for every $t \in[a, b]$, the sequences $\left(\alpha^{1}(t), \ldots, \alpha^{m}(t)\right)$ and $\left(\beta^{1}(t), \ldots, \beta^{m}(t)\right)$ are equal after removing all 0's.

Then $\alpha=\beta$.

Proof Induction with respect to $m$. For $m=1$ this is obvious. Let $s \in[a, b]$ be the maximal number such that $\alpha^{j}(s)=0$ for some $j \in\{1, \ldots, n\}$. Condition (c) implies that $\alpha(t)=\beta(t)$ for $t>s$, since there are no 0's to remove. By continuity also $\alpha(s)=\beta(s)$. If $s=0$, then the lemma is proven. Assume $s>0$ and let $A=\left\{j \mid \alpha^{j}(s)=\beta^{j}(s)=0\right\}$. Let $\alpha^{\prime}, \beta^{\prime} \in \vec{P}_{[a, s]}\left(\vec{I}^{m-|A|}\right)$ be the paths obtained from $\left.\alpha\right|_{[a, s]}$ and $\left.\beta\right|_{[a, s]}$ by removing all coordinates belonging to $A$. By the inductive hypothesis, we have $\alpha^{\prime}=\beta^{\prime}$ and, therefore, $\left.\alpha\right|_{[a, s]}=\left.\beta\right|_{[a, s]}$.

Proposition 8.7 Every regular natural tame d-path $\alpha \in \vec{N}_{[0, n]}^{t}(K)_{\mathbf{0}}^{\mathbf{1}}$ admits a unique minimal nt-presentation, which is regular. As a consequence, all nt-presentations of $\alpha$ are equivalent.

Proof Let $\alpha=[\mathbf{c} ; \beta]$ be a regular nt-presentation and let $\alpha=\left[\mathbf{c}^{\prime} ; \beta^{\prime}\right]$ be a minimal presentation. By Proposition 8.5, we have $\operatorname{Vert}\left(\mathbf{n}^{\mathbf{c}}\right)=\operatorname{Vert}(\alpha)=\operatorname{Vert}\left(\mathbf{n}^{\mathbf{c}^{\prime}}\right)$ and hence $\mathbf{n}^{\mathbf{c}}=\mathbf{n}^{\mathbf{c}^{\prime}}$. Fix $i \in\left\{1, \ldots, l(\mathbf{c})=l\left(\mathbf{c}^{\prime}\right)\right\}$ and choose $t \in\left[t_{i-1}^{\mathbf{c}}, t_{i}^{\mathbf{c}}\right]$ such that $\beta_{i}(t) \in$ $\vec{I}^{n_{i}^{\mathrm{c}}} \backslash \partial \vec{I}^{n_{i}^{\mathrm{c}}}$. Now $\left[c_{i} ; \beta_{i}(t)\right]$ is a canonical presentation of the point $\alpha(i)$. Since $\operatorname{dim}\left(c_{i}^{\prime}\right)=$ $\operatorname{dim}\left(c_{i}\right)$, also $\left[c_{i}^{\prime}, \beta_{i}^{\prime}(t)\right]$ is a canonical presentation of $\alpha(t)$, which implies that $c_{i}=c_{i}^{\prime}$ and $\beta_{i}(t)=\beta_{i}^{\prime}(t)$. Applying Lemma 8.6 we obtain that $\left.\beta_{i}\right|_{\left[t_{i-1}^{\mathbf{c}}, t\right]}=\left.\beta_{i}^{\prime}\right|_{\left[t_{i-1}^{\mathbf{c}}, t\right]}$, and the "opposite" analogue of this lemma implies that $\left.\beta_{i}\right|_{\left[t, t_{i}^{\mathbf{c}}\right]}=\left.\beta_{i}^{\prime}\right|_{\left[t, t_{i}^{\mathbf{c}}\right]}$. As a consequence, $c_{i}=c_{i}^{\prime}$ and $\beta_{i}=\beta_{i}^{\prime}$ for all $i$, and hence $\mathbf{c}=\mathbf{c}^{\prime}$ and $\beta=\beta^{\prime}$. Thus, the minimal nt-presentation of $\alpha$ is unique and all nt-presentations of $\alpha$ are equivalent to it.

Proposition 8.8 Assume that $K \subseteq \square^{n}$. Then every natural tame path $\alpha \in \vec{N}_{[0, n]}^{t}(K)_{\mathbf{0}}^{\mathbf{1}}$ admits a unique minimal presentation.

Proof Let $\alpha=[\mathbf{c}, \beta]$ and $\alpha=\left[\mathbf{c}^{\prime}, \beta^{\prime}\right]$ be minimal nt-presentations. We have $\mathbf{n}:=$ $\mathbf{n}^{\mathbf{c}}=\mathbf{n}^{\mathbf{c}^{\prime}}$. For every $i \in\left\{1, \ldots, l(\mathbf{c})=l\left(\mathbf{c}^{\prime}\right)\right\}$ we have

$$
\begin{aligned}
& {\left[d^{0}\left(c_{i}^{\prime}\right) ;()\right]=\left[c_{i}^{\prime} ; \mathbf{0}\right]=\left[c_{i}^{\prime}, \beta_{i}^{\prime}\left(t_{i-1}^{\mathbf{n}}\right)\right]=\alpha\left(t_{i-1}^{\mathbf{n}}\right)=\left[c_{i}, \beta_{i}\left(t_{i-1}^{\mathbf{n}}\right)\right]=\left[c_{i}, \mathbf{0}\right]=\left[d^{0}\left(c_{i}\right) ;()\right]} \\
& {\left[d^{1}\left(c_{i}^{\prime}\right) ;()\right]=\left[c_{i}^{\prime} ; \mathbf{1}\right]=\left[c_{i}^{\prime}, \beta_{i}^{\prime}\left(t_{i}^{\mathbf{n}}\right)\right]=\alpha\left(t_{i}^{\mathbf{n}}\right)=\left[c_{i}, \beta_{i}\left(t_{i}^{\mathbf{n}}\right)\right]=\left[c_{i}, \mathbf{1}\right]=\left[d^{1}\left(c_{i}\right) ;()\right] .}
\end{aligned}
$$

Thus, $c_{i}=c_{i}^{\prime}$ since they have the same extreme vertices. Furthermore, all the maps $\left|c_{i}\right|: \vec{I}^{n_{i}} \rightarrow \vec{I}^{n}$ are injective, which shows that $\beta_{i}=\beta_{i}^{\prime}$. 


\section{Comparison of the colimit and the space of natural tame d-paths}

Fix a bi-pointed $\square$-set $K$ and an integer $n \geq 0$. In this Section we investigate the sequence of surjective maps

$$
\coprod_{\mathbf{c} \in \operatorname{Ch}(K ; n)} \vec{N}_{[0, n]}\left(\vec{I}^{\vee \mathbf{n}^{\mathbf{c}}}\right)_{\mathbf{0}}^{\mathbf{1}} \rightarrow \underset{\mathbf{c} \in \operatorname{Ch}(K ; n)}{\operatorname{colim}} \vec{N}_{[0, n]}\left(\vec{I}^{\vee \mathbf{n}^{\mathbf{c}}}\right)_{\mathbf{0}}^{\mathbf{1}} \stackrel{F_{K}^{n}}{\longrightarrow} \vec{N}_{[0, n]}^{t}(K)_{\mathbf{0}}^{\mathbf{1}}
$$

The spaces appearing in this sequence are, respectively from left to right, the space of nt-presentations, the space of equivalence classes of nt-presentations and the space of natural tame d-paths. The main goal of this section is to prove that the map $F_{K}^{n}$ is a homotopy equivalence. Recall that $\operatorname{Tam}_{n}^{K}$ is the tamification map defined in (6.9).

Proposition 9.1 Let $\alpha \in \vec{N}_{[0, n]}^{t}(K)_{\mathbf{0}}^{\mathbf{1}}$. Assume that $\operatorname{Vert}(\alpha)=\operatorname{Vert}\left(\operatorname{Tam}_{n}^{K}(\alpha)\right)$. Then $\alpha$ is a regular natural tame d-path.

Proof Let $\alpha=[\mathbf{c} ; \beta]$ be a minimal nt-presentation. Then also

$$
\operatorname{Tam}_{n}^{K}(\alpha)=\left[\mathbf{c} ; \operatorname{Tam}_{n}^{\square^{\vee \mathbf{n}}}(\beta)\right]
$$

is a minimal nt-presentation, since $\operatorname{Tam}_{n}$ is functorial, and

$$
\operatorname{Vert}\left(\operatorname{Tam}_{n}^{K}(\alpha)\right)=\operatorname{Vert}(\alpha)=\operatorname{Vert}\left(\mathbf{n}^{\mathbf{c}}\right)
$$

Assume that $\alpha$ is not regular. Thus, there exists $i \in\{1, \ldots, l(\mathbf{c})\}$ such that $\beta_{i} \in$ $\vec{N}_{\left[t_{i-1}^{\mathbf{c}}, t_{i}^{\mathbf{c}}\right]}\left(\partial \vec{I}^{n_{i}^{\mathbf{c}}}\right)_{\mathbf{0}}^{\mathbf{1}}$. Then

$$
\operatorname{Tam}_{n}^{\square^{\vee n^{\mathbf{c}}}}(\beta)_{i}:=\left.\operatorname{Tam}_{n}^{\square^{\vee \mathbf{n}}}(\beta)\right|_{\left[t_{i-1}^{\mathbf{c}}, t_{i}^{\mathbf{c}}\right]} \in \vec{N}_{\left[t_{i-1}^{\mathbf{c}}, t_{i}^{\mathbf{c}}\right]}\left(\partial \vec{I}^{n_{i}^{\mathbf{c}}}\right)_{\mathbf{0}}^{\mathbf{1}}
$$

is tame (regarded as a d-path in $\partial \vec{I}^{n_{i}^{\mathbf{c}}}$ ) and, therefore, $k \in \operatorname{Vert}\left(\operatorname{Tam}_{n}^{\square^{\vee \mathbf{n}^{\mathbf{c}}}}\left(\beta_{i}\right)\right.$ ) for some $t_{i-1}^{\mathbf{c}}<k<t_{i}^{\mathbf{c}}$. This contradicts the assumption, since $k \notin \operatorname{Vert}(\alpha)$.

Proposition 9.2 Let $\alpha \in \vec{N}_{[0, n]}^{t}(K)_{\mathbf{0}}^{\mathbf{1}}$. If $\alpha=[\mathbf{c} ; \beta]$ and $\alpha=\left[\mathbf{c}^{\prime} ; \beta^{\prime}\right]$ are equivalent nt-presentations, then also $\operatorname{Tam}_{n}^{K}(\alpha)=\left[\mathbf{c} ; \operatorname{Tam}_{n}^{\square^{\vee \mathbf{n}}}(\beta)\right]$ and $\operatorname{Tam}_{n}^{K}(\alpha)=$ $\left[\mathbf{c}^{\prime} ; \operatorname{Tam}_{n}^{\square^{\vee \mathbf{n}^{\prime}}}\left(\beta^{\prime}\right)\right]$ are equivalent nt-presentations.

Proof This follows from the functoriality of $\operatorname{Tam}_{n}$.

Below we change decorations at Tam; the upper index in $\mathrm{Tam}^{m}$ stands for the $m$-fold composition.

Proposition 9.3 Assume that $m \geq n$. Let $\alpha \in \vec{N}_{[0, n]}^{t}(K)_{0}^{\mathbf{1}}$ and let $\alpha=[\mathbf{c} ; \beta]$ and $\alpha=$ $\left[\mathbf{c}^{\prime} ; \beta^{\prime}\right]$ be nt-presentations of $\alpha$. Then the nt-presentations $\operatorname{Tam}^{m}(\alpha)=\left[\mathbf{c} ; \operatorname{Tam}^{m}(\beta)\right]$ and $\operatorname{Tam}^{m}(\alpha)=\left[\mathbf{c}^{\prime} ; \operatorname{Tam}^{m}\left(\beta^{\prime}\right)\right]$ are equivalent. 
Proof We have an ascending sequence of sets

$$
\begin{aligned}
\{0, n\} & \subseteq \operatorname{Vert}(\alpha) \subseteq \operatorname{Vert}(\operatorname{Tam}(\alpha)) \\
& \subseteq \operatorname{Vert}\left(\operatorname{Tam}^{2}(\alpha)\right) \subseteq \cdots \subseteq \operatorname{Vert}\left(\operatorname{Tam}^{m}(\alpha)\right) \subseteq\{0, \ldots, n\}
\end{aligned}
$$

Hence, there exists $r \in\{0, \ldots, m-1\}$ such that $\operatorname{Vert}\left(\operatorname{Tam}^{r}(\alpha)\right)=\operatorname{Vert}\left(\operatorname{Tam}^{r+1}(\alpha)\right)$. By Proposition 9.1, $\operatorname{Tam}^{r}(\alpha)$ is a regular tame path and hence, by Proposition 8.7, the nt-presentations $\operatorname{Tam}^{r}(\alpha)=\left[\mathbf{c} ; \operatorname{Tam}^{r}(\beta)\right]$ and $\operatorname{Tam}^{r}(\alpha)=\left[\mathbf{c}^{\prime} ; \operatorname{Tam}^{r}\left(\beta^{\prime}\right)\right]$ are equivalent. Now the conclusion follows from Proposition 9.2.

In terms of diagrams, Proposition 9.3 states that for every $m \geq n$ in the diagram of solid arrows

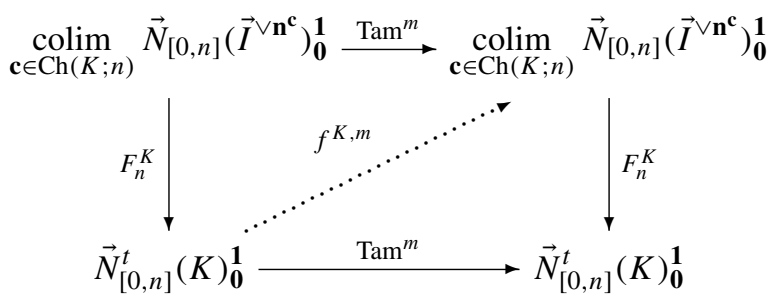

there exists a function $f^{K, m}$ that makes the upper triangle commutative. Since $F_{n}^{K}$ is surjective, also the lower triangle commutes. However, it is not clear whether $f^{K, m}$ is continuous. We will tackle with this problem in the rest of this section, starting with the following lemma.

Proposition 9.4 Let $X, Y$ be metric spaces and let $L>0$. Let $\operatorname{Lip}_{L}(X, Y)$ denote the space of L-Lipschitz maps $X \rightarrow Y$ with compact-open topology and let $Y^{X}$ be the space of all continuous maps $X \rightarrow Y$ with the product topology. Assume that $Y$ is compact. Then the inclusion map $\operatorname{Lip}_{L}(X, Y) \stackrel{\subseteq}{\rightarrow} Y^{X}$ is a homeomorphism on its image.

Proof We need to prove the following statement: for every compact subset $K \subseteq X$, an open subset $U \subseteq Y$ and $f \in \operatorname{Lip}_{L}(X, Y)$ such that $f(K) \subseteq U$ there exist sequences $x_{1}, \ldots, x_{n}$ of points of $X$ and $U_{1}, \ldots, U_{n}$ of open subsets of $Y$ such that

$$
\forall_{g \in \operatorname{Lip}_{L}(X, Y)}\left(\left(\forall_{i=1}^{n} g\left(x_{i}\right) \in U_{i}\right) \Rightarrow g(K) \subseteq U\right) .
$$

Since $f(K)$ is compact, the distance $d$ between $f(K)$ and $Y \backslash U$ is strictly positive. Let $\left\{x_{1}, \ldots, x_{n}\right\}$ be a family of points of $K$ such that the family of open balls having radius $d / 2 L$ and centered at the $x_{i}$ 's cover $K$. Let $U_{i}=B\left(f\left(x_{i}\right), d / 2\right)$. Assume that $g \in \operatorname{Lip}_{L}(X, Y)$ is a function such that $g\left(x_{i}\right) \in U_{i}$ for all $i$. For every $x \in K$ there exists $i \in\{1, \ldots, n\}$ such that $\operatorname{dist}_{X}\left(x, x_{i}\right)<d / 2 L$. Therefore,

$$
\begin{aligned}
\operatorname{dist}_{Y}\left(g(x), f\left(x_{i}\right)\right) & \leq \operatorname{dist}_{Y}\left(g(x), g\left(x_{i}\right)\right)+\operatorname{dist}_{Y}\left(g\left(x_{i}\right), f\left(x_{i}\right)\right) \\
& \leq L \operatorname{dist}_{X}\left(x, x_{i}\right)+d / 2 \leq d .
\end{aligned}
$$


As a consequence, $g(x) \in U$, which ends the proof.

Proposition 9.5 For every $\mathbf{n} \in \operatorname{Seq}(n)$, the space $\vec{N}_{[0, n]}\left(\vec{I}^{\vee \mathbf{n}}\right)_{\mathbf{0}}^{\mathbf{1}}$ is compact.

Proof Since

$$
\vec{N}_{[0, n]}\left(\vec{I}^{\vee \mathbf{n}}\right)_{\mathbf{0}}^{\mathbf{1}} \cong \prod_{i=1}^{l(\mathbf{n})} \vec{N}_{\left[t_{i-1}^{\mathbf{n}}, t_{i}^{\mathbf{n}}\right]}\left(\vec{I}^{n_{i}}\right)_{\mathbf{0}}^{\mathbf{1}},
$$

it is sufficient to prove that $\vec{N}_{[0, n]}\left(\vec{I}^{n}\right)_{\mathbf{0}}^{\mathbf{1}}$ is compact. But $\vec{N}_{[0, n]}\left(\vec{I}^{n}\right)_{\mathbf{0}}^{\mathbf{1}}$ is a closed subset of $\operatorname{Lip}_{1}\left(I, I^{n}\right)$, with $L^{1}$-metric on $I^{n}$, so the statement follows from Proposition 9.4.

Proposition 9.6 If $K$ is finite, then the map $f^{K, m}$ (9.2) is continuous for $m \geq n$. For arbitrary $K, f^{K, m}$ is continuous for $m \geq n+1$.

Proof If $K$ is finite, then so is $\operatorname{Ch}(K ; n)$. Thus, $\underset{\mathbf{c} \in \operatorname{Ch}(K ; n)}{\operatorname{colim}} \vec{N}_{[0, n]}\left(\vec{I}^{\vee \mathbf{n}^{\mathbf{c}}}\right)_{\mathbf{0}}^{\mathbf{1}}$ is compact by Proposition 9.5 and then the left-hand vertical map is a quotient map. As a consequence, $f_{m}$ is continuous for $m \geq n$.

Assume that $m \geq n+1$. For any d-path $\alpha \in \vec{N}_{[0, n]}^{t}(K)_{\mathbf{0}}^{\mathbf{1}}$ there exists a finite sub- $\square$-set $L \subseteq K$ such that $\alpha \in \vec{N}_{[0, n]}(L)_{\mathbf{0}}^{\mathbf{1}}$. Let

$$
U_{L}=\left\{x \in|K| \mid \operatorname{dist}(x,|L|)<\frac{1}{4}\right\}
$$

where dist stands for the $L_{1}$-distance on $|K|$. The set $\vec{N}_{[0, n]}^{t}\left(U_{L}\right)_{\mathbf{0}}^{\mathbf{1}}$ is an open neighborhood of $\alpha$, which is mapped by Tam into $\vec{N}_{[0, n]}^{t}(L)_{\mathbf{0}}^{\mathbf{1}}$; this follows from properties (a) and (b) of $R$. The restriction of $f^{K, m}$ to $\vec{N}_{[0, n]}^{t}(U)_{0}^{\mathbf{1}}$ is equal to the composition of continuous maps

$\vec{N}_{[0, n]}^{t}(U)_{\mathbf{0}}^{\mathbf{1}} \stackrel{\operatorname{Tam}}{\longrightarrow} \vec{N}_{[0, n]}^{t}(L)_{\mathbf{0}}^{\mathbf{1}} \stackrel{f^{L, m-1}}{\longrightarrow} \underset{\mathbf{c} \in \operatorname{Ch}(L ; n)}{\operatorname{colim}} \vec{N}_{[0, n]}\left(\vec{I}^{\vee \mathbf{n}^{\mathbf{c}}}\right)_{\mathbf{0}}^{\mathbf{1}} \subseteq \underset{\mathbf{c} \in \operatorname{Ch}(K ; n)}{\operatorname{colim}} \vec{N}_{[0, n]}\left(\vec{I}^{\vee \mathbf{n}^{\mathbf{c}}}\right)_{\mathbf{0}}^{\mathbf{1}}$

Thus, $f^{K, m}$ it is continuous at every point (d-path) $\alpha$.

As a consequence, for every $m \geq n+1$ (9.2) is a commutative diagram of continuous maps. Since the horizontal maps are homotopic to the identities on the respective spaces, we obtain:

Proposition 9.7 The map

$$
F_{n}^{K}: \underset{\mathbf{c} \in \operatorname{Ch}_{n}(K)_{\mathbf{0}}^{\mathbf{1}}}{\operatorname{colim}} \vec{N}_{[0, n]}\left(\vec{I}^{\vee \mathbf{n}^{\mathbf{c}}}\right)_{\mathbf{0}}^{\mathbf{1}} \rightarrow \vec{N}_{[0, n]}^{t}(K)_{\mathbf{0}}^{\mathbf{1}}
$$

is a homotopy equivalence with a homotopy inverse $f_{n+1}^{K}$. 


\section{Comparison of the homotopy colimit with the colimit}

Fix a bi-pointed $\square$-set $K$ and an integer $n \geq 0$.

In this section we show that the map

$$
Q_{n}^{K}: \underset{\mathbf{c} \in \operatorname{Ch}(K ; n)}{\operatorname{hocolim}} \vec{N}\left(\square^{\vee \mathbf{n}^{\mathbf{c}}}\right)_{\mathbf{0}}^{\mathbf{1}} \rightarrow \underset{\mathbf{c} \in \operatorname{Coh}(K ; n)}{\operatorname{colim}} \vec{N}\left(\square^{\vee \mathbf{n}^{\mathbf{c}}}\right)_{\mathbf{0}}^{\mathbf{1}}
$$

is a weak homotopy equivalence. We will use the following criterion due to Dugger (2008). Let $\mathcal{C}$ be an upwards-directed Reedy category (Dugger 2008, Definition 13.6) and let $F: \mathcal{C} \rightarrow$ Top be a diagram. The latching object of $c \in \mathrm{Ob}(\mathcal{C})$ is

$$
L_{c} F:=\operatorname{colim}_{\partial \mathcal{C} \downarrow c} F \circ \text { dom }
$$

where $\partial \mathcal{C} \downarrow c$ is the slice category over the object $c$ with the identity object id $_{\mathbf{c}}$ removed, and dom : $\mathcal{C} \downarrow c \rightarrow \mathcal{C}$ is the forgetful functor. The latching object is equipped with the latching map $\Lambda_{c}: L_{c} F \rightarrow F(c)$ that is induced by the cocone $\{F(\varphi)\}_{(\varphi: a \rightarrow c) \in \partial \mathcal{C} \downarrow c}$.

Proposition 10.1 (Dugger 2008, Proposition 14.2) Assume that the latching map $\Lambda_{c}$ is a cofibration for every object $c \in \mathrm{Ob}(\mathcal{C})$. Then $F$ is projective-cofibrant and so $\operatorname{hocolim}_{\mathcal{C}} F \rightarrow \operatorname{colim}_{\mathcal{C}} F$ is a weak equivalence.

The category $\operatorname{Ch}(K ; n)$ admits a grading $\operatorname{deg}(\mathbf{a})=n-l(\mathbf{a})$, which makes it an upwards-directed Reedy category: for every non-identity morphism $\varphi: \mathbf{a} \rightarrow \mathbf{b}$ in $\mathrm{Ch}(K)$ we have $l(\mathbf{a})>l(\mathbf{b})$.

For every $\mathbf{c} \in \mathrm{Ch}(K ; n)$, the functor

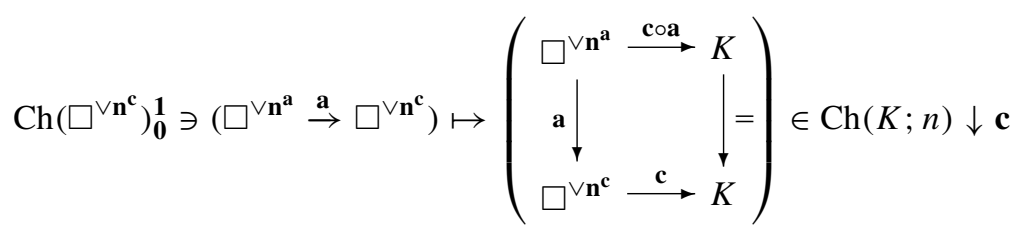

is an isomorphism of categories: the functor dom : $\mathrm{Ch}(K ; n) \downarrow \mathbf{c} \rightarrow \square \mathrm{Ch}_{n}$ is its inverse. Furthermore, the functors $\vec{N}_{[0, n]}\left(\square^{\vee \mathbf{n}^{(-)}}\right)_{\mathbf{0}}^{\mathbf{1}}$ on $\mathrm{Ch}\left(\square^{\vee \mathbf{n}^{\mathbf{c}}}\right)$ and $\vec{N}_{[0, n]}\left(\square^{\vee \mathbf{n}^{(-)}}\right)_{\mathbf{0}}^{\mathbf{1}}$ 。 dom on $\mathrm{Ch}(K ; n) \downarrow \mathbf{c}$ are naturally equivalent. As a consequence, the latching map $L_{\mathbf{c}} \vec{N}_{[0, n]}\left(\vec{I}^{\vee \mathbf{n}^{(-)}}\right) \rightarrow \vec{N}_{[0, n]}\left(\square^{\vee \mathbf{n}^{\mathbf{c}}}\right)$ is related by homeomorphisms to the canonical map

$$
\underset{\mathbf{a} \in \partial \operatorname{Ch}\left(\square^{\vee \mathbf{n}^{\mathbf{c}}}\right)}{\operatorname{colim}} \vec{N}_{[0, n]}\left(\vec{I}^{\vee \mathbf{n}^{\mathbf{a}}}\right)_{\mathbf{0}}^{\mathbf{1}} \rightarrow \vec{N}_{[0, n]}\left(\vec{I}^{\vee \mathbf{n}^{\mathbf{c}}}\right)_{\mathbf{0}}^{\mathbf{1}},
$$

where $\partial \mathrm{Ch}\left(\square^{\vee \mathbf{n}^{\mathbf{c}}}\right)$ is the full subcategory of $\mathrm{Ch}\left(\square^{\vee \mathbf{n}^{\mathbf{c}}}\right)$ with the identity cube chain $\square^{\vee \mathbf{n}^{\mathbf{c}}} \stackrel{=}{\rightarrow} \square^{\vee \mathbf{n}^{\mathbf{c}}}$ removed. In particular, the latching map of $\mathbf{c}$ depends only on the type $\mathbf{n}^{\mathbf{c}}$ of $\mathbf{c}$.

For $\mathbf{n} \in \operatorname{Seq}(n)$ denote

$$
\partial \vec{N}_{[0, n]}\left(\square^{\vee \mathbf{n}}\right)_{\mathbf{0}}^{\mathbf{1}}=\left\{\alpha \in \vec{N}_{[0, n]}\left(\square^{\vee \mathbf{n}}\right)_{\mathbf{0}}^{\mathbf{1}} \mid \operatorname{Vert}(\alpha) \backslash \operatorname{Vert}(\mathbf{n}) \neq \emptyset\right\} ;
$$


this is the space of all natural tame paths that admit an nt-presentation $[\mathbf{c} ; \beta]$ such that $\mathbf{c}$ is a non-identity cube chain, i.e., $\mathbf{c} \neq \mathrm{id}_{\square^{\vee \mathbf{n}}}$.

Proposition 10.2 For every $\mathbf{n} \in \operatorname{Seq}(n)$ we have

$$
\operatorname{im}\left(\underset{\mathbf{c} \in \partial \operatorname{Ch}\left(\square^{\vee \mathbf{n}}\right)}{\operatorname{colim}} \vec{N}_{[0, n]}\left(\vec{I}^{\vee \mathbf{n}^{\mathbf{c}}}\right)_{\mathbf{0}}^{\mathbf{1}} \stackrel{\Lambda_{\mathbf{n}}}{\longrightarrow} \vec{N}_{[0, n]}\left(\vec{I}^{\vee \mathbf{n}}\right)\right)=\partial \vec{N}_{[0, n]}\left(\square^{\vee \mathbf{n}}\right)_{\mathbf{0}}^{\mathbf{1}}
$$

Furthermore, $\Lambda_{\mathbf{n}}$ is a homeomorphism onto its image.

Proof Assume that $\alpha \in \partial \vec{N}_{[0, n]}\left(\square^{\vee \mathbf{n}}\right)_{\mathbf{0}}^{\mathbf{1}}$ and let $\alpha=[\mathbf{c} ; \beta]$ be a minimal ntpresentation, which exists due to Proposition 8.4. Since $\operatorname{Vert}\left(\mathbf{n}^{\mathbf{c}}\right)=\operatorname{Vert}(\alpha) \neq$ $\operatorname{Vert}(\mathbf{n}), \mathbf{c}$ is not the identity cube chain. Therefore, $\Lambda_{n}(\mathbf{c}, \beta)=\alpha$, which implies that $\partial \vec{N}_{[0, n]}\left(\square^{\vee \mathbf{n}}\right)_{\mathbf{0}}^{\mathbf{1}} \subseteq \operatorname{im}\left(\Lambda_{\mathbf{n}}\right)$.

If $\alpha \in \operatorname{im}\left(\Lambda_{\mathbf{n}}\right)$, then there exists an nt-presentation $\alpha=[\mathbf{c}, \beta]$ such that $\mathbf{c} \neq \mathrm{id}_{\square} \vee \mathbf{n}$. Thus, $\operatorname{Vert}(\alpha) \supseteq \operatorname{Vert}\left(\mathbf{n}^{\mathbf{c}}\right) \supsetneq \operatorname{Vert}(\mathbf{n})$ and, therefore, $\alpha \in \partial \vec{N}_{[0, n]}\left(\square^{\vee \mathbf{n}}\right)_{\mathbf{0}}^{\mathbf{1}}$.

There exists an embedding of $\square^{\vee \mathbf{n}}$ in $\square^{n}$. Then Proposition 8.8 implies that all nt-presentations of a d-path $\alpha \in \partial \vec{N}_{[0, n]}\left(\square^{\vee \mathbf{n}}\right)_{\mathbf{0}}^{\mathbf{1}}$ are equivalent, since they are all equivalent to a unique minimal nt-presentation. As a consequence, $\left(\Lambda_{\mathbf{n}}\right)^{-1}(\alpha)$ is a one-point set and, therefore, $\Lambda_{\mathbf{n}}$ is a bijection onto its image.

By Proposition 9.5, all the spaces appearing in the colimit are compact. Since the indexing category $\partial \mathrm{Ch}\left(\square^{\vee \mathbf{n}}\right)$ is finite, the colimit is compact and $\Lambda_{\mathbf{n}}$ is a homeomorphism onto its image.

Proposition 10.3 For every $\mathbf{n} \in \operatorname{Seq}(n)$, the inclusion $\partial \vec{N}_{[0, n]}\left(\vec{I}^{\vee \mathbf{n}}\right)_{\mathbf{0}}^{\mathbf{1}} \subset \vec{N}_{[0, n]}\left(\vec{I}^{\vee \mathbf{n}}\right)_{\mathbf{0}}^{\mathbf{1}}$ is a cofibration.

Proof Denote for short $\partial \vec{N}:=\partial \vec{N}_{[0, n]}\left(\square^{\vee \mathbf{n}}\right)_{\mathbf{0}}^{\mathbf{1}}, \vec{N}:=\vec{N}_{[0, n]}\left(\square^{\vee \mathbf{n}}\right)_{\mathbf{0}}^{\mathbf{1}}, \vec{P}:=$ $\vec{P}_{[0, n]}\left(\square^{\vee \mathbf{n}}\right)_{\mathbf{0}}^{\mathbf{1}}$. We will prove that $\partial \vec{N}$ is a strong neighborhood deformation retract in $\vec{N}$. If $\mathbf{n}=(1, \ldots, 1)$, then $\operatorname{Vert}(\mathbf{n})=\{0,1, \ldots, n\}$ and hence $\partial \vec{N}=\emptyset$. Assume that $\mathbf{n} \neq(1, \ldots, 1)$ and fix an embedding $\square^{\vee \mathbf{n}} \subseteq \square^{n}$. For any d-path $\alpha \in \vec{P}_{[0, n]}\left(\vec{I}^{\vee \mathbf{n}}\right)_{\mathbf{0}}^{\mathbf{1}}$, let $\alpha^{j} \in \vec{P}_{[0, n]}(\vec{I})_{0}^{1}$ denote the $j$ th coordinate of $\alpha$, regarded as a d-path in $\vec{I}^{n}$ (via the chosen embedding $\left.\vec{I}^{\mathbf{n}}=\left|\square^{\vee \mathbf{n}}\right| \subseteq\left|\square^{n}\right|=\vec{I}^{n}\right)$.

$\operatorname{Denote} \operatorname{Free}(\mathbf{n})=\{1, \ldots, n-1\} \backslash \operatorname{Vert}(\mathbf{n})$. The function

$$
f: \vec{N} \ni \alpha \mapsto \min _{k \in \operatorname{Free}(\mathbf{n})} \max _{j \in\{1, \ldots, n\}} \min \left\{\alpha^{j}(k), 1-\alpha^{j}(k)\right\} \in[0,1]
$$

is continuous, and $f^{-1}(0)=\partial \vec{N}$. Fix $0<\varepsilon<\frac{1}{n}$ and let $U=f^{-1}([0, \varepsilon))$; we will construct a strong deformation retraction of $U$ into $\partial \vec{N}$.

For $a \in[0, \varepsilon]$ let $g(a,-): \vec{I} \rightarrow \vec{I}$ be the function such that $g(a, h)=0$ for $h \in[0, a], g(a, t)=1$ for $h \in[1-a, 1]$ and $g(a,-)$ is linear on the interval $[a, 1-a]$. Note that $|t-g(a, t)| \leq a$ for all $t \in \vec{I}$.

For $s \in[0,1], \alpha \in \vec{N}$ and $t \in[0, n]$ let

$$
G(s, \alpha)(t)=(1-s) \alpha(t)+s\left(g\left(f(\alpha), \alpha^{1}(t)\right), g\left(f(\alpha), \alpha^{2}(t)\right), \ldots, g\left(f(\alpha), \alpha^{n}(t)\right)\right) .
$$


If $\alpha^{j}(t)=\eta$ for $\eta \in\{0,1\}$, then $g\left(f(\alpha), \alpha^{j}(t)\right)=\eta$, which implies that $G(s, \alpha)(t) \in$ $\vec{I}^{\vee \mathbf{n}}$ for all $s \in[0,1]$. Thus, this formula defines a continuous map $G:[0,1] \times U \rightarrow \vec{P}$. Let us prove that the composition nat $\circ G$ is a strong deformation retraction of $U$ into $\partial \vec{N}$. Indeed, for every $\alpha \in \partial \vec{N}$ we have $f(\alpha)=0$ and, therefore, $\operatorname{nat}(G(s, \alpha))=$ nat $(\alpha)=\alpha$ for all $s \in[0,1]$. If $\alpha \in U$, then there exists $k \in$ Free(n) such that

$$
\min \left\{\alpha^{j}(k), 1-\alpha^{j}(k)\right\} \leq f(\alpha)
$$

for all $j \in\{1, \ldots, n\}$, i.e., $\alpha^{j}(k) \in[0, f(\alpha)] \cup[1-f(\alpha), 1]$. Thus,

$$
G(1, \alpha)(k)=\left(g\left(f(\alpha), \alpha^{1}(k)\right), \ldots, g\left(f(\alpha), \alpha^{n}(k)\right)\right)
$$

is a vertex. Furthermore,

$$
\begin{aligned}
\left|k-\operatorname{len}\left(\left.G(1, \alpha)\right|_{[0, k]}\right)\right| & =\left|k-\sum_{j=1}^{n} g\left(f(\alpha), \alpha^{j}(k)\right)\right| \\
& =\left|\sum_{j=1}^{n} \alpha^{j}(k)-\sum_{j=1}^{n} g\left(f(\alpha), \alpha^{j}(k)\right)\right| \\
& \leq \sum_{j=1}^{n}\left|\alpha^{j}(k)-g\left(f(\alpha), \alpha^{j}(k)\right)\right| \leq n f(\alpha)<1 .
\end{aligned}
$$

Thus, $\operatorname{len}\left(\left.G(1, \alpha)\right|_{[0, k]}\right)=k$ and hence nat $(G(1, \alpha))(k)=G(1, \alpha)(k)$ is a vertex, which shows that nat $\circ G(1, \alpha) \in \partial \vec{N}$.

Proposition 10.4 The map

$$
Q_{n}^{K}: \underset{\mathbf{c} \in \operatorname{Ch}(K ; n)}{\operatorname{hocolim}} \vec{N}\left(\square^{\vee \mathbf{n}^{\mathbf{c}}}\right)_{\mathbf{0}}^{\mathbf{1}} \rightarrow \underset{\mathbf{c} \in \operatorname{Ch}(K ; n)}{\operatorname{colim}} \vec{N}\left(\square^{\vee \mathbf{n}^{\mathbf{c}}}\right)_{\mathbf{0}}^{\mathbf{1}}
$$

is a weak homotopy equivalence.

Proof By Propositions 10.2 and 10.3, the assumptions of Proposition 10.1 are satisfied.

\section{Compliance with ethical standards}

Conflict of interest The author states that there is no conflict of interest.

Open Access This article is distributed under the terms of the Creative Commons Attribution 4.0 International License (http://creativecommons.org/licenses/by/4.0/), which permits unrestricted use, distribution, and reproduction in any medium, provided you give appropriate credit to the original author(s) and the source, provide a link to the Creative Commons license, and indicate if changes were made. 


\section{References}

Dubut, L., Goubault, E., Goubault-Larrecq, L.: Natural homology. In: Halldórsson, M., Iwama, K., Kobayashi, N., Speckmann, B. (eds.) Automata, Languages, and Programming. ICALP 2015. Lecture Notes in Computer Science, vol. 9135. Springer, Berlin (2015)

Dugger, D.: A primer on homotopy colimits (2008). Preprint https://pages.uoregon.edu/ddugger/hocolim. pdf

Fahrenberg, U., Legay, A.: History-preserving bisimilarity for higher-dimensional automata via open maps. Electron. Notes Theor. Comput. Sci. 298, 165-178 (2013)

Fajstrup, L.: Dipaths and dihomotopies in a cubical complex. Adv. Appl. Math. 35, 188-206 (2005)

Fajstrup, L., Goubault, E., Raussen, M.: Algebraic topology and concurrency. Theor. Comput. Sci. 357, 241-278 (2006)

Fajstrup, L., Goubault, E., Haucourt, E., Mimram, S., Raussen, M.: Directed Algebraic Topology and Concurrency. Springer, Berlin (2016)

Goubault, E.: Labelled cubical sets and asynchronous transition systems: an adjunction (2002). http://www. lix.polytechnique.fr/ goubault/papers/cmcim02.pdf

Grandis, M.: Directed homotopy theory, I. The fundamental category. Cah. Topol. Geom. Differ. Categ. 44, 281-316 (2003)

Pratt, V.: Modelling concurrency with geometry. In: Proceedings of the 18th ACM Symposium on Principles of Programming Languages, pp. 311-322 (1991)

Raussen, M.: Invariants of directed spaces. Appl. Categ. Stuct. 15, 355-386 (2007)

Raussen, M.: Trace spaces in a pre-cubical complex. Topol. Appl. 156, 1718-1728 (2009)

Raussen, M.: Simplicial models for trace spaces. Algebr. Geom. Topol. 10, 1683-1714 (2010)

Raussen, M.: Simplicial models for trace spaces II: general higher dimensional automata. Algebr. Geom. Topol. 12, 1545-1565 (2012)

Raussen, M., Ziemiański, K.: Homology of spaces of directed paths on Euclidian cubical complexes. J. Homotopy Relat. Struct. 9, 67-84 (2014)

van Glabbeek, R.J.: On the expressiveness of higher dimensional automata. Theor. Comput. Sci. 368, 168-194 (2006)

Ziemiański, K.: A cubical model for path spaces in d-simplicial complexes. Topol. Appl. 159, 2127-2145 (2012). https://doi.org/10.1016/j.topol.2012.02.005

Ziemiański, K.: Spaces of directed paths on pre-cubical sets. Appl. Algebra Eng. Commun. Comput. 28, 497-525 (2017). https://doi.org/10.1007/s00200-017-0316-0

Ziemiański, K.: Directed path spaces via discrete vector fields. Appl. Algebra Eng. Commun. Comput. 30, 51-74 (2019a). https://doi.org/10.1007/s00200-018-0360-4

Ziemiański, K.: Stable components of directed spaces. Appl. Categ. Struct. 27, 217-244 (2019b). https:// doi.org/10.1007/s10485-018-9551-1

Publisher's Note Springer Nature remains neutral with regard to jurisdictional claims in published maps and institutional affiliations. 